ARTICLE

\title{
High-pH structure of EmrE reveals the mechanism of proton-coupled substrate transport
}

\author{
Alexander A. Shcherbakov (1) ${ }^{1}$, Peyton J. Spreacker (10 2 , Aurelio J. Dregni (1) ${ }^{1}$, \\ Katherine A. Henzler-Wildman (i) ${ }^{2} \&$ Mei Hong (i) ${ }^{1 凶}$
}

The homo-dimeric bacterial membrane protein EmrE effluxes polyaromatic cationic substrates in a proton-coupled manner to cause multidrug resistance. We recently determined the structure of substrate-bound EmrE in phospholipid bilayers by measuring hundreds of protein-ligand $\mathrm{H}^{\mathrm{N}}-\mathrm{F}$ distances for a fluorinated substrate, 4-fluoro-tetraphenylphosphonium $\left(\mathrm{F}_{4}-\mathrm{TPP}^{+}\right)$, using solid-state NMR. This structure was solved at low $\mathrm{pH}$ where one of the two proton-binding Glu14 residues is protonated. Here, to understand how substrate transport depends on $\mathrm{pH}$, we determine the structure of the EmrE-TPP complex at high $\mathrm{pH}$, where both Glu14 residues are deprotonated. The high-pH complex exhibits an elongated and hydrated binding pocket in which the substrate is similarly exposed to the two sides of the membrane. In contrast, the low-pH complex asymmetrically exposes the substrate to one side of the membrane. These $\mathrm{pH}$-dependent EmrE conformations provide detailed insights into the alternating-access model, and suggest that the high-pH conformation may facilitate proton binding in the presence of the substrate, thus accelerating the conformational change of EmrE to export the substrate.

\footnotetext{
${ }^{1}$ Department of Chemistry, Massachusetts Institute of Technology, 170 Albany Street, Cambridge, MA 02139, USA. ${ }^{2}$ Department of Biochemistry,

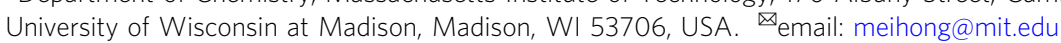


$\mathrm{M}$ ultidrug resistance (MDR) is a major public health concern that can undermine years of drug development efforts and result in epidemics of drug-resistant infections ${ }^{1}$. One of the mechanisms by which cells can become resistant to therapeutics is via expression of transmembrane (TM) efflux pump proteins in the small multidrug resistance (SMR) family. These SMR transporters remove cytotoxins from the cytoplasm by coupling the uphill efflux process to the downhill influx of protons across the cytoplasmic membrane (Fig. la) ${ }^{2}$. Unlike selective active transport proteins that recognize and transport a single substrate, the SMR proteins efflux a variety of cytotoxic compounds with different shapes, sizes, and chemical properties ${ }^{3,4}$. The relatively small size of these transporters was originally thought to provide a minimal model system for studying secondary active transport ${ }^{5}$. However, due to the sensitivity of these proteins to their environment, their conformational plasticity, and lack of extracellular domains, high-resolution structural information had been limited for many years ${ }^{6-10}$. The best studied SMR transporter, EmrE, is involved in $\mathrm{pH}$ and osmotic stress responses, biofilm formation, and resistance to a variety of polyaromatic cations ${ }^{11-14}$. Biochemical and biophysical data have shown that the transport process of EmrE is highly complex. For example, in addition to acting as a proton-coupled antiporter, EmrE can also function as a proton-coupled symporter or uncoupled uniporter under different conditions ${ }^{15-19}$. Elucidating the mechanism of membrane transport by EmrE requires atomicresolution structural information for multiple states of the protein, as well as dynamics information about the protein and the ligands throughout the transport cycle.

The 110-residue EmrE forms an antiparallel, asymmetric homodimer. Early electron microscopy (EM) data established that EmrE did not possess two-fold symmetry9,20. A subsequent $3.8 \AA$ crystal structure showed that the two subunits are oriented in an antiparallel fashion, indicating that the protein has dual membrane topology ${ }^{8}$. This antiparallel conformation was later confirmed by NMR and single-molecule FRET data ${ }^{21}$, mutagenesis ${ }^{22-24}$, EPR data ${ }^{25,26}$, and studies of homologous proteins ${ }^{10,27}$. Solution and solid-state NMR (ssNMR) data showed that the two subunits of the dimer are conformationally asymmetric, exhibiting two sets of chemical shifts, and the TM helices undergo major reorientations as they exchange between the inward- and outward-facing states $6,16,21,28,29$. Crosslinking the antiparallel dimer blocked alternating acces in vitro and ethidium resistance in vivo, demonstrating the functional importance of this structural transition ${ }^{29}$. The proton-binding residue of EmrE is E14, which exhibits $\mathrm{pK}_{a}$ values of $7.2 \pm 0.1$ and $8.4 \pm 0.2$ in the $\mathrm{A}$ and $\mathrm{B}$ subunits of the dimer in both lipid bilayers and bicelles ${ }^{15,30}$. Solution NMR experiments showed that the substrate, tetraphenylphosphonium $\left(\mathrm{TPP}^{+}\right)$, binds the protein asymmetrically, interacting primarily with one subunit and protecting that E14 from protonation, while E14 in the other subunit remains accessible to protonation with a $\mathrm{pK}_{a}$ of $6.8^{15,17}$. This proton-binding asymmetry



Fig. 1 Schematic of the EmrE transport function. a Simplified mechanistic model of $\mathrm{pH}$-dependent substrate transport by EmrE. The binding-site structure of the cationic substrate in the dimeric protein depends on the protonation state of the proton-binding residue E14. $\mathbf{b}$ Structure of the substrate $\mathrm{F}_{4}-\mathrm{TPP}^{+}$. was confirmed by magic-angle-spinning (MAS) NMR data that show that the E14 sidechain carboxyl chemical shifts differ between the two subunits ${ }^{31}$. A $2.3 \AA$ crystal structure of the EmrE homolog, Gdx, was determined in complex with monobodies ${ }^{10}$. This structure confirms the asymmetry of the antiparallel dimer structure and the asymmetric interaction of the substrate with the key glutamate residues in the pore. However, Gdx is a selective guanidinium efflux pump, not a multidrug transporter-like EmrE, and biophysical data is more limited for the Gdx transporter. To fully understand the molecular mechanism of EmrE transport, high-resolution structures are essential. This experimental structure information will enable an integrated analysis of the wealth of data on EmrE dynamics and function, allow assessment of the validity of molecular dynamics simulations $s^{32,33}$ performed using the backbone-only EmrE crystal structure, and give insight into the similarities and differences between selective and non-selective transporters in the SMR family.

Recently, we discovered a single-point mutant, S64V-EmrE, that retains wild-type substrate-binding affinity but has slower rates of alternating access ${ }^{7}$. At the same time, we developed a ${ }^{1} \mathrm{H}-{ }^{19} \mathrm{~F}$ REDOR NMR technique to measure distances to the $\sim 2 \mathrm{~nm}$ range for structure determination ${ }^{34-38}$. Due to ${ }^{1} \mathrm{H}$ detection under fast MAS, this technique has high spectral sensitivity, thus increasing the throughput of the distance measurement. Exploiting these biochemical and spectroscopic advances, we determined an experimental structure of S64V-EmrE complexed to a fluorinated substrate, $\mathrm{F}_{4}$-TPP ${ }^{+}$(Fig. 1b) in DMPC bilayers at $\mathrm{pH} 5.8^{39}$. At this acidic $\mathrm{pH}$, one of the two E14 residues is protonated and neutral while the other residue remains deprotonated and anionic. By measuring 200 protein-ligand distances as well as site-specific protein chemical shifts, we determined the structure of this acidic-pH complex (abbreviated as EmrE-TPP below) to an average pairwise backbone root-mean-square deviation (RMSD) of $1.6 \AA$. The structure was calculated by docking the ligand to the protein, followed by molecular dynamics simulations that equilibrate the protein in explicitly solvated lipid bilayers, all under experimentally measured distance and torsion angle constraints. This low-pH structure shows that the cationic substrate lies closer to E14A than E14B, consistent with the asymmetric $\mathrm{pK}_{a}$ 's. The binding pocket is lined with numerous aromatic residues, including W63, Y60, F44, and Y40. These aromatic sidechains interact with the four ligand phenylene rings to stabilize the substrate, while still leaving sufficient space for the substrate to reorient. While this low-pH structure of EmrE gives a glimpse of the protein-substrate binding geometry, it is not sufficient for revealing the transport mechanism, because alternating access requires the protein to adopt multiple conformations throughout the transport cycle. Experimental data on multiple structural states is required to understand how drug binding and proton binding drive the conformational changes needed to transport the substrates across the membrane.

Here we determine the high-pH structure of the EmrE-TPP complex using the ${ }^{1} \mathrm{H}-{ }^{19} \mathrm{~F}$ REDOR NMR experiment. We measure $\sim 380$ protein-ligand $\mathrm{H}^{\mathrm{N}}-\mathrm{F}$ distances, which combine with chemical-shift derived torsion angles to enable the calculation of the high-pH structure. We also investigate millisecond-timescale motion of $\mathrm{F}_{4}-\mathrm{TPP}^{+}$at the binding site using $2 \mathrm{D}^{19} \mathrm{~F}-{ }^{19} \mathrm{~F}$ exchange NMR. These structural and dynamical results provide information about how changes in the protonation state of the protein drives structural transitions that enable EmrE to transport substrates in a proton-coupled manner.

\section{Results}

Resonance assignment of the high-pH EmrE-TPP complex. To understand how proton binding and release change the structure of substrate-bound EmrE, we first ascertained the $\mathrm{E} 14 \mathrm{pK}_{a}$ of 
S64V-EmrE in complex with $\mathrm{F}_{4}-\mathrm{TPP}^{+}$. We previously carried out extensive $\mathrm{pH}$ titrations of $\mathrm{TPP}^{+}$-bound EmrE in bicelles using solution NMR and found that the WT transporter had a single $\mathrm{pK}_{a}$ of $6.8 \pm 0.1^{17}$, while S64V-EmrE had a single $\mathrm{pK}_{a}$ of $7.0 \pm 0.1^{7}$. We verified that this $\mathrm{pK}_{a}$ was not significantly different when $\mathrm{S} 64 \mathrm{~V}$-EmrE is bound to $\mathrm{F}_{4}-\mathrm{TPP}^{+}$by repeating this $\mathrm{pH}$ titration. Global analysis of multiple residues yields a $\mathrm{pK}_{a}$ value of $6.9 \pm 0.1$ (Supplementary Fig. 1). We therefore prepared a sample of S64V-EmrE bound to $\mathrm{F}_{4}-\mathrm{TPP}^{+}$in DMPC bilayers at $\mathrm{pH} 8.0$ to determine the structure of the unprotonated complex, to compare with the protonated complex previously determined at $\mathrm{pH} 5.8$ in DMPC bilayers.

We first assessed the conformational homogeneity of the high$\mathrm{pH}$ EmrE-TPP complex using $2 \mathrm{D}{ }^{1} \mathrm{H}-{ }^{15} \mathrm{~N}$ correlation (hNH) spectra (Fig. 2a). The spectra show a typical ${ }^{15} \mathrm{~N}$ linewidth of $\sim 0.5$ ppm, which is narrower than the low-pH sample ${ }^{39}$, indicating that the protein is structurally more homogeneous at high $\mathrm{pH}$ than at low $\mathrm{pH}$. The ${ }^{15} \mathrm{~N}$ and ${ }^{1} \mathrm{H}$ chemical shifts at high $\mathrm{pH}$ differ moderately from the low- $\mathrm{pH}$ values, which preclude the transfer of the chemical shifts from the low-pH spectra. Thus, we measured eight $3 \mathrm{D}^{1} \mathrm{H}$-detected correlation spectra (Fig. 2b, c and Supplementary Table 1) to independently assign the resonances of the high-pH complex. Among these experiments, the 3D hNcacoNH and HncacoHN spectra are particularly useful for sequence-specific assignment. Based on the peak connectivities, we obtained four backbone chemical shifts $\left(\mathrm{Ca}, \mathrm{CO}, \mathrm{N}^{\mathrm{H}}, \mathrm{H} N\right)$ for 77 residues in subunit $A$ and 64 residues in subunit $B$. In addition, 64 residues in subunit $A$ and 53 residues in subunit $B$ have assigned $\mathrm{C} \beta$ chemical shifts. The resulting secondary chemical shifts confirm the presence of four TM helices in the protein (Supplementary Fig. 2). However, the high-pH EmrE exhibits significant chemical shift differences from the low-pH protein in the TM3 and TM1 helices of subunit B (Supplementary Fig. 3). These two TM helices contain the important functional residues W63 and E14, respectively, suggesting that the protein interacts with the substrate differently between high and low $\mathrm{pH}$. Between subunits A and B, TM1 and TM3 helices have larger chemical shift differences compared to TM2 and TM4 helices, similarly indicating the importance of TM1 and TM3 helices for ligand binding (Supplementary Fig. 4).

Measurement of protein-ligand $\mathbf{H}^{\mathrm{N}}-\mathbf{F}$ distances. The assignment of the $\mathrm{H}^{\mathrm{N}}$ and ${ }^{15} \mathrm{~N}$ chemical shifts allowed us to measure and resolve protein-substrate $\mathrm{H}^{\mathrm{N}}-\mathrm{F}$ distances using the ${ }^{1} \mathrm{H}$-detected and $\mathrm{hNH}$-resolved ${ }^{1} \mathrm{H}-{ }^{19} \mathrm{~F}$ REDOR experiment ${ }^{37}$. We measured the 2D REDOR spectra at three mixing times, 1.68, 2.53 , and $3.78 \mathrm{~ms}$. For each mixing time, a control $2 \mathrm{D} S_{0}$ spectrum with ${ }^{19} \mathrm{~F}$ pulses off and a dephased $S$ spectrum with ${ }^{19} \mathrm{~F}$ pulses on were measured. The former exhibits all backbone and sidechain $\mathrm{H}^{\mathrm{N}}$ signals (Fig. 3a) whereas the latter shows weaker intensities for amide protons that experience significant ${ }^{1} \mathrm{H}-{ }^{19} \mathrm{~F}$ dipolar couplings. The difference spectrum $(\Delta S)$ between $S_{0}$ and $S$ thus manifests only the signals of amide protons that are near the fluorines. At $1.68 \mathrm{~ms}$, we observed signals only from the nearest residues to $\mathrm{TPP}^{+}$, such as E14, Y40, Y60, and W63, whereas at longer mixing times, signals from more remote residues such as G9, G57, and S75 are also detected. No difference signals are observed for residues C-terminal to the TM3 helix and for loop residues.

Fitting the intensity ratios $S / S_{0}$ between the REDOR $S_{0}$ and $S$ spectra allowed us to extract precise $\mathrm{H}^{\mathrm{N}}-\mathrm{F}$ distances (Fig. $3 \mathrm{~b}$ and Supplementary Fig. 5). We observed significant dipolar dephasing for many residues. Among 116 resolved dipolar dephasing curves, 36 show REDOR dephasing that corresponds to distances of less than $9 \AA$. The shortest distance is found for W63B indole $\mathrm{N} \varepsilon$, which is $3.8 \AA$ from the nearest fluorine. In general, TM2 and TM3 residues have some of the closest contact with $\mathrm{F}_{4}-\mathrm{TPP}^{+}$. For example, the $\mathrm{S} 43 \mathrm{~A} \mathrm{H} \mathrm{H}^{\mathrm{N}}$ is $4.3 \AA$ from the nearest ${ }^{19} \mathrm{~F}$. The distances in the high-pH complex differ substantially from the low-pH values. For example, A10B, S43B, and $\mathrm{W} 63 \mathrm{~B} \varepsilon$ are closer to the ligand fluorines by $1.2-2.5 \AA$ while $\mathrm{T} 18 \mathrm{~A}$ and $\mathrm{W} 63 \mathrm{~A}$ backbone amides are further from the ligand fluorines by $0.2-0.3 \AA$.

Ligand docking and structure calculation. Using rigid-body docking (Supplementary Fig. 6a), we disambiguated the four-fold degeneracy of the fluorines and assigned the measured dipolar couplings to specific protein amide protons (Supplementary Tables 2 and 3). For weak REDOR dephasing that corresponds to distances longer than $10 \AA$, four constraints were created where the protein $\mathrm{H}^{\mathrm{N}}$ atom must be at least $10 \AA$ away from each of the four fluorines. In total, we obtained 387 protein-ligand distance restraints from this docking analysis (Supplementary Table 4). $\mathrm{F}_{4^{-}}$ $\mathrm{TPP}^{+}$docks to a single location between the two subunits, analogous to the low-pH complex. But the ligand orientation relative to the protein differs substantially from the low- $\mathrm{pH}$ structure. At high $\mathrm{pH}$, three of the four phenylene ring planes are approximately parallel to the bilayer normal. In comparison, at low $\mathrm{pH}$, only one phenylene ring is tangential to the bilayer normal whereas three rings lie transverse to the bilayer normal (Fig. $4 \mathrm{a}$, b). Using two lowest-violation docked structures, we refined the protein structure using molecular dynamics simulations under the protein-ligand distance constraints, 148 pairs of $(\phi, \psi)$ angles and $76 \chi_{1}$ torsion angles (Table 1 and Supplementary Table 5). The simulations equilibrated by $200 \mathrm{~ns}$ to a backbone RMSD of $2.85 \pm 0.95 \AA$ for the protein (Supplementary Fig. 6b) and $1.30 \pm 0.64 \AA$ for the $\mathrm{F}_{4}-\mathrm{TPP}^{+}$phosphorous and its four directly bonded carbons (Table 1). Among the four TM helices, TM4 is the furthest away from the ligand: all resolved dipolar couplings correspond to distances of longer than $9 \AA$ (Supplementary Fig. 5). This is consistent with the low-resolution crystal structure, which shows that TM1-TM3 form the substrate-binding pocket whereas the TM4 helices that control dimerization are away from the transport pore ${ }^{8}$. Since TM4 is not well constrained by the measured distances (Supplementary Fig. 6c), when we consider only the TM1-3 helices, the calculated structure has an improved backbone RMSD of $1.97 \pm 0.67 \AA$.

Structural differences between the high-pH and low-pH complex. Interestingly, the high-pH EmrE-TPP complex exhibits numerous structural differences from the low-pH complex (Fig. 4 and Supplementary Fig. 7). First, the ligand is more symmetrically positioned between the two E14 residues at high $\mathrm{pH}$ : the distances between the $\mathrm{F}_{4}-\mathrm{TPP}^{+}$phosphorus $(\mathrm{P})$ and the two E14 C $\delta$ carbons are $6.6 \pm 0.7 \AA$ to subunit $\mathrm{A}$ and $7.5 \pm 0.5 \AA$ to subunit B. In comparison, at low $\mathrm{pH}$ the $\mathrm{P}$ atom is $1.9 \AA$ closer to the negatively charged E14A than to the neutral E14B (Table 2 and Fig. 4e, f). Thus, the protonation states of the two E14 residues directly impact the substrate position. Second, the inter-subunit proximities and relative orientations of the TM helices have changed between the high-pH and low-pH complexes. At high $\mathrm{pH}$, the two E14-bearing TM1 helices are further away from each other, while the two W63-bearing TM3 helices are more parallel to each other (Fig. 4a, b and Supplementary Fig. 7) compared to the low-pH complex. The E14A CA-E14B CA distance is $16.9 \pm 0.6 \AA$ at high $\mathrm{pH}$ and shortens to $15.7 \pm 0.8 \AA$ at low $\mathrm{pH}$. Overall, the TM helices become more parallel to each other at high $\mathrm{pH}$, creating an elongated binding cavity in which $\mathrm{F}_{4}-\mathrm{TPP}^{+}$is oriented with three 

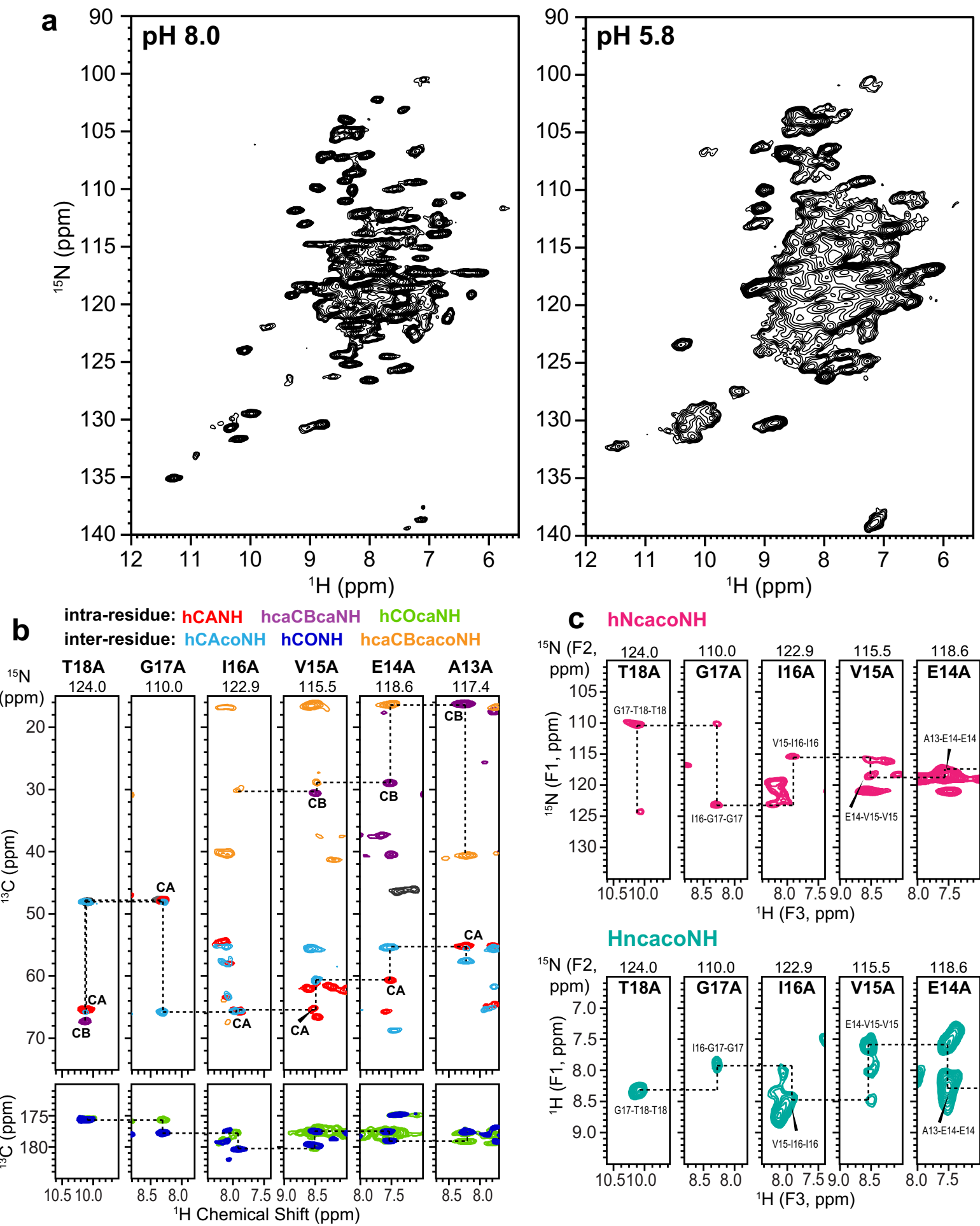

\section{C hNcacoNH}

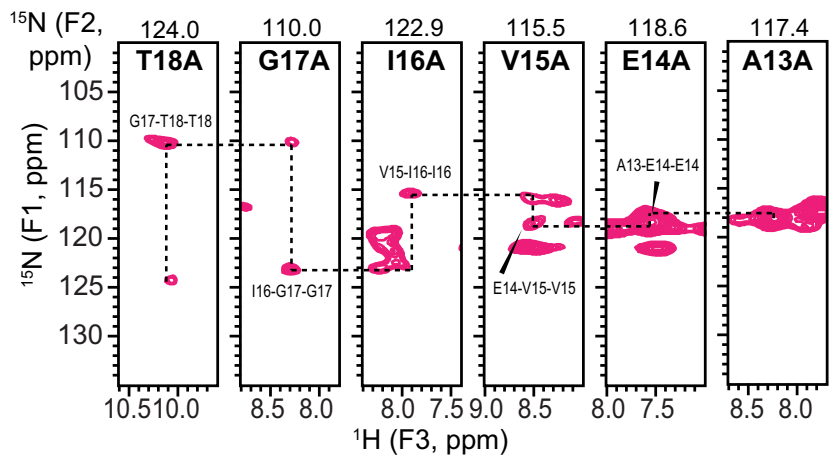

HncacoNH

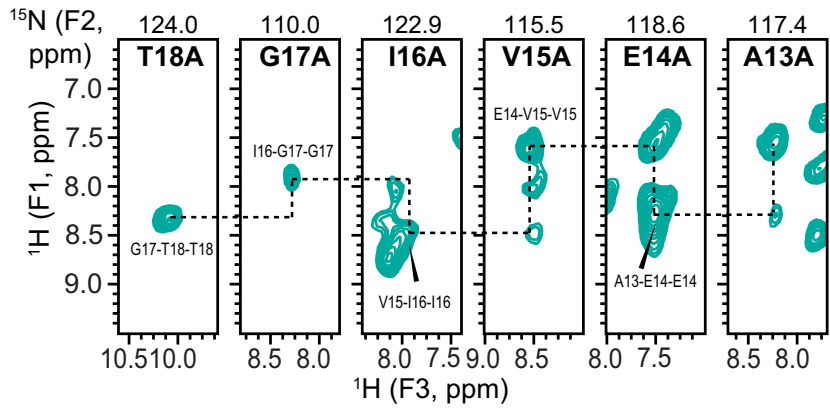

Fig. 2 2D and 3D ${ }^{1} \mathbf{H}$-detected MAS correlation spectra of TPP-bound EmrE in DMPC bilayers for resonance assignment. a 2D hNH spectra of the protein at $\mathrm{pH} 8.0$ compared to $\mathrm{pH} 5.8$. The spectral linewidths are narrower at high $\mathrm{pH}$, indicating higher structural homogeneity. $\mathbf{b}$ Six ${ }^{1} \mathrm{H}-{ }^{13} \mathrm{C}-{ }^{15} \mathrm{~N} 3 \mathrm{D}$ correlation spectra to obtain intra-residue and inter-residue peak connectivities. The strips for residues T18 to $\mathrm{A} 13$ in subunit $\mathrm{A}$ are shown. The aliphatic ${ }^{13} \mathrm{C}$ strips overlay four 3D spectra whereas the carbonyl ${ }^{13} \mathrm{C}$ strips overlay two 3D spectra. Negative intensities in the hcaCBcaNH and hcaCBcacoNH spectra are shown in gray. Glycine residues show a negative CA peak rather than a positive CB peak. c Inter-residue 3D NNH and HNH correlation spectra. All spectra were measured under $55 \mathrm{kHz}$ MAS on a $600 \mathrm{MHz}$ NMR spectrometer.

out of four phenylene rings tangential to the bilayer normal. In comparison, at low $\mathrm{pH}$ the TM helices are oriented at very different angles with respect to each other. In particular, the $\mathrm{N}$-terminal end of TM3A approaches the C-terminal end of TM3B (Supplementary Fig. 7b), which closes off water access on one side of the helical bundle. This closed-on-one-side configuration pushes the ligand towards the opposite end of the helical bundle, where the C-terminal end of TM3A is now splayed open from the N-terminal end of TM3B. The resulting shallow and open binding site at low $\mathrm{pH}$ exposes the ligand (Supplementary 

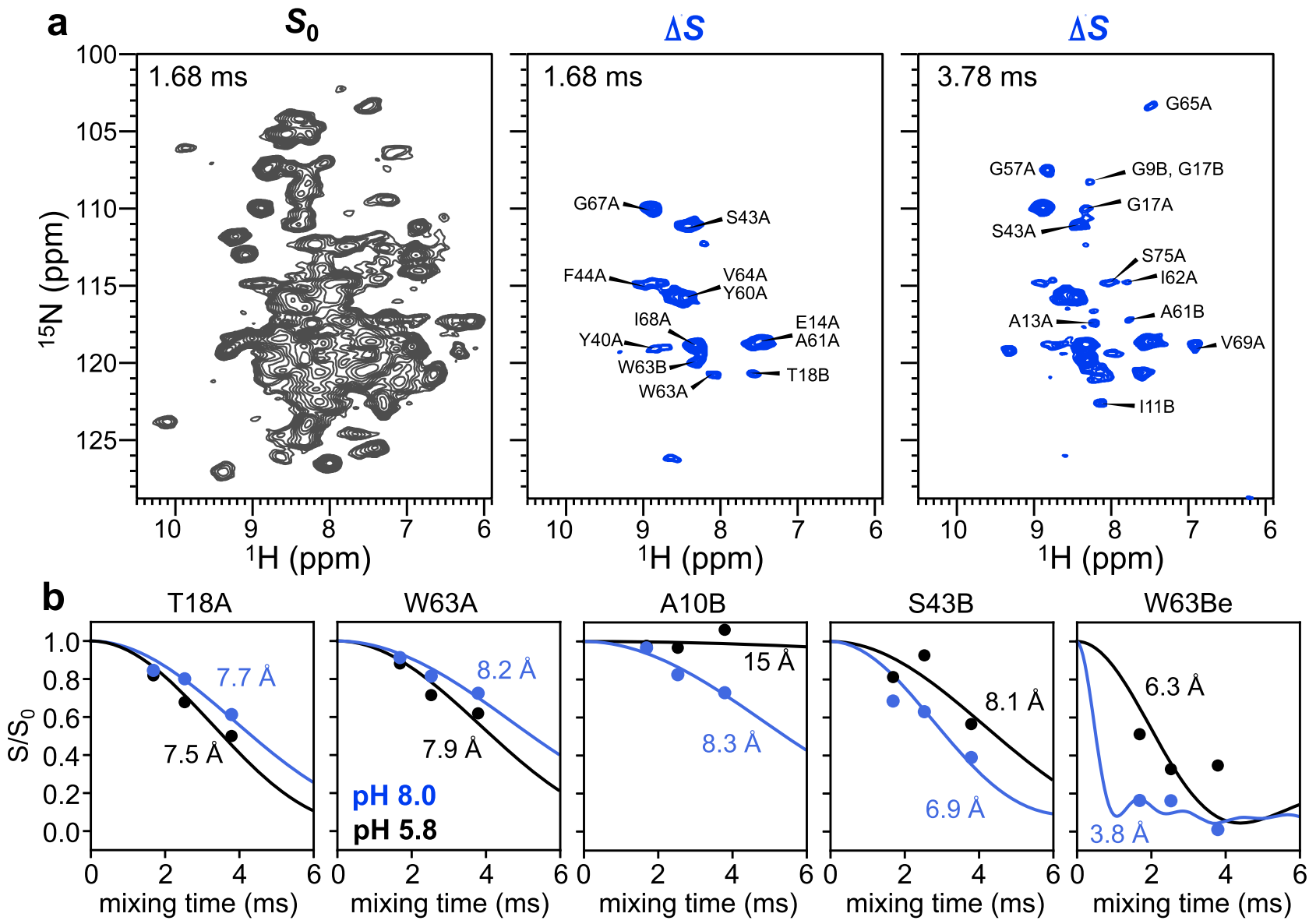

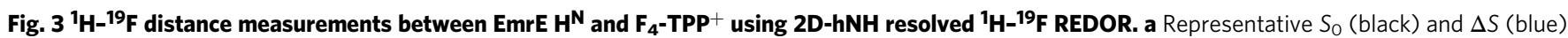
spectra for two mixing times, 1.68 and $3.78 \mathrm{~ms}$. Assignment is shown for peaks in the $\Delta S$ spectrum. As the mixing time increases, more difference peaks are observed, corresponding to $\mathrm{H}^{\mathrm{N}}$ sites that are further from the fluorinated substrate. b Representative ${ }^{1} \mathrm{H}-19 \mathrm{~F}$ REDOR $\mathrm{S} / \mathrm{S}_{\mathrm{O}}$ dephasing curves with best-fit simulations. ${ }^{1} \mathrm{H}-{ }^{19} \mathrm{~F}$ distances at $\mathrm{pH} 8.0$ (blue) differ from the $\mathrm{pH} 5.8$ data (black). The indole $\mathrm{H}^{\mathrm{N}}$ of $\mathrm{W}^{2} 3 \mathrm{~B}$ has much shorter distances to the substrate fluorines at $\mathrm{pH} 8.0$ than at $\mathrm{pH}$ 5.8. REDOR dephasing values are provided as a Source Data file.

Fig. $7 \mathrm{~b}$ ), allowing three of the four phenylene rings to lie transverse to the bilayer normal.

Compared to the low-pH complex, the high-pH EmrE-TPP complex has a longer and more symmetric binding cavity that is not fully closed on either side. Because the binding pocket is populated by protein sidechains, we next investigated which binding site is more spacious by measuring substrate dynamics using ${ }^{19} \mathrm{~F}$ NMR. This is a more direct and functional probe of the binding-site volume compared to computing the volume based on the structure. One-dimensional ${ }^{19} \mathrm{~F}$ direct-polarization (DP) NMR spectra of the substrate at high $\mathrm{pH}$ resolve three peaks with intensity ratios of 1:1:2 (Fig. 5a), indicating that the four fluorines of the ligand reside in distinct chemical and conformational environments. The most downfield peak (peak 4) has a narrow linewidth of $0.8 \mathrm{ppm}(\sim 450 \mathrm{~Hz})$, indicating that one of the fluorines resides in a well-ordered structural environment. The presence of one narrow ${ }^{19} \mathrm{~F}$ peak is also observed at low $\mathrm{pH}$, but this narrow peak has the most upfield chemical shift. In both cases, ${ }^{1} \mathrm{H}-{ }^{19} \mathrm{~F}$ cross-polarization (CP) spectra preferentially enhanced the intensity of the narrow peak relative to the other ${ }^{19} \mathrm{~F}$ signals, indicating that this fluorine is the most immobilized. Compared to the low-pH sample, the high- $\mathrm{pH}^{19} \mathrm{~F}$ chemical shifts of $\mathrm{F}_{4}-\mathrm{TPP}^{+}$shifted by about $6 \mathrm{ppm}$ downfield, indicating that the binding-site aromatic residues interact with the substrate very differently at high $\mathrm{pH}$.
Dynamics and hydration of the ligand at the binding site. To probe millisecond-timescale motions of the ligand in the binding pocket, we measured $2 \mathrm{D}{ }^{19} \mathrm{~F}-{ }^{19} \mathrm{~F}$ exchange spectra of $\mathrm{F}_{4}-\mathrm{TPP}^{+}$at $285 \mathrm{~K}$ using mixing times of $0.1 \mathrm{~ms}$ to $80 \mathrm{~ms}$ (Fig. 5b). Exchange cross peaks are readily observed by $\sim 20 \mathrm{~ms}$ at this temperature, but are absent at $265 \mathrm{~K}^{39}$, indicating that exchange on this timescale is due to motion rather than ${ }^{19} \mathrm{~F}$ spin diffusion. The diagonal intensity decays and cross-peak intensity buildup occur with rates of $165-318 s^{-1}$ (Fig. $5 c$ and Supplementary Fig. 8). These rates are about two-fold faster than the low-pH rates $\left(75-103 \mathrm{~s}^{-1}\right)$, indicating that the ligand is more dynamic in the high-pH complex. These increased dynamics at high $\mathrm{pH}$ agree well with the measured thermodynamic parameters for ligand binding. EmrE is a protoncoupled transporter, and we have previously shown that protons are released from both E14 and $\mathrm{H} 110$ upon $\mathrm{TPP}^{+}$-binding ${ }^{18}$. ITC experiments were performed using multiple buffers with different ionization enthalpies to determine the number of protons released. These experiments were performed at four different $\mathrm{pH}$ values. This data was analyzed to extract the enthalpy and entropy of binding independent of the buffer contribution by extrapolationg to $\Delta H_{\text {ionization }}=0$ (Supplementary Fig. 9). The resulting thermodynamic parameters are shown in Table 3. This data shows that the well-established increase in binding affinity at high $\mathrm{pH}$ is driven by an increasingly favorable entropic contribution, while the enthalpy of binding becomes less favorable. 


\section{a $\quad \mathrm{pH} 8.0$}

Top view (periplasmic side)

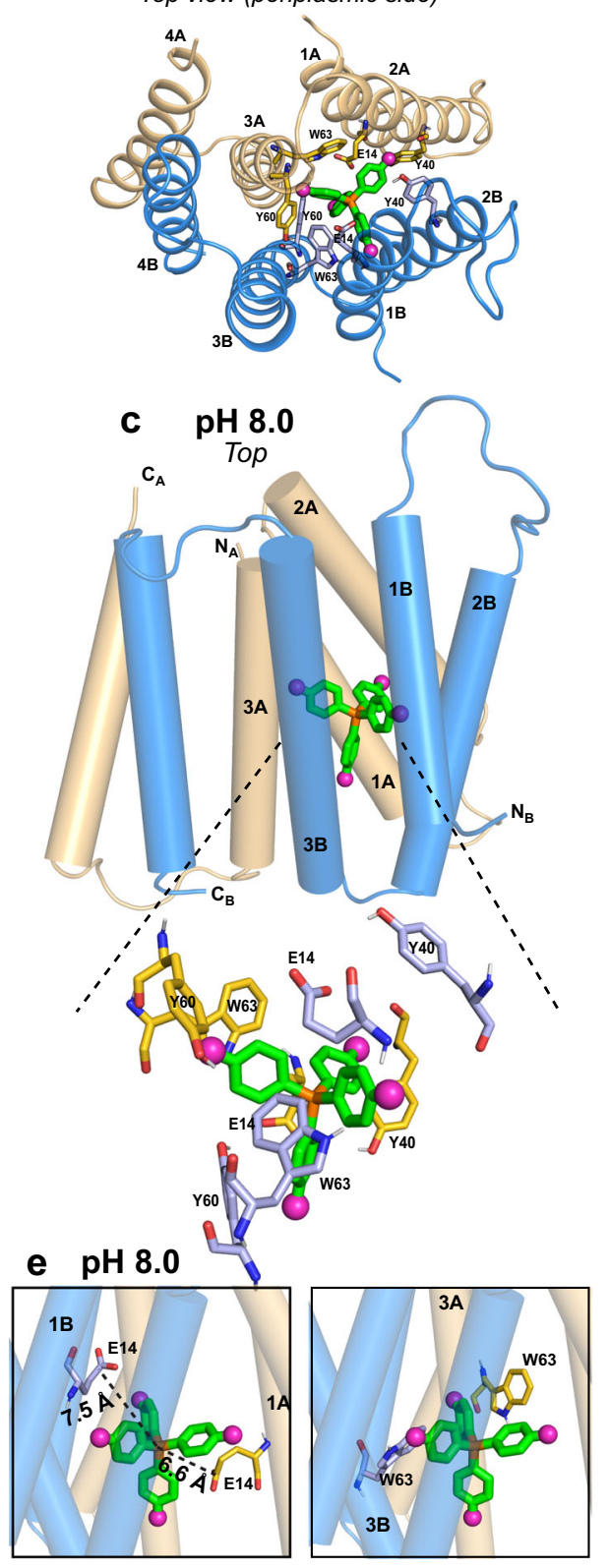

b $\quad$ pH 5.8
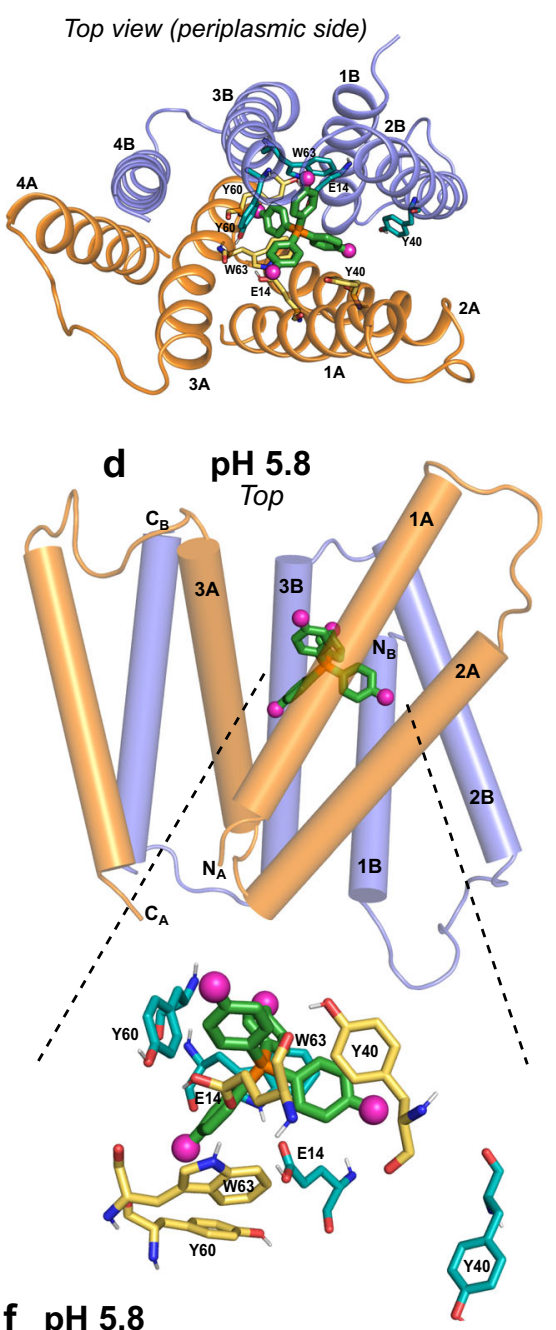

f $\mathrm{pH} 5.8$


Fig. 4 Structure of the EmrE-TPP complex in DMPC bilayers at high pH (PDB: 7SFQ) and its comparison with the previously determined low-pH structure (PDB: 7JK8). a Top view of the high-pH EmrE-TPP complex structure, seen from the periplasmic side. The crucial binding-site residues E14, Y40, Y60, and W63 are shown as sticks. b Top view of the low-pH EmrE-TPP complex for comparison. Note that conformer A (beige) in the high-pH complex has changed to conformer B (purple) in the low-pH complex. This conformational change switches the designation of the two subunits between the high and low $\mathrm{pH}$ complexes. c Side view of the high-pH structure. $\mathbf{d}$ Side view of the low-pH structure. The ligand is buried deep in the high-pH complex but is more exposed to the top side in the low-pH complex. The arrangement of the TM helices differs noticeably between the two structures. e TPP+ position relative to E14 and W63 in the high-pH structure. The ligand center $\mathrm{P}$ atom is similarly distanced from the two E14 residues. $\mathbf{f}$ TPP position relative to E14 and W63 in the low-pH structure. The ligand is $2 \AA$ closer to E14A than E14B.

The faster ligand reorientations in the high-pH complex suggest that the binding pocket should be more hydrated compared to the low-pH complex. To test this hypothesis, we measured water-edited 2D hNH spectra of the protein using water-to-protein ${ }^{1} \mathrm{H}$ polarization transfer times of 30 and $325 \mathrm{~ms}$ (Fig. 6). The short-mixing-time spectra selectively exhibit wellhydrated residues. After correcting for ${ }^{1} \mathrm{H} \quad \mathrm{R}_{1}$ relaxation (Supplementary Fig. 10), we find that the high-pH complex exhibits higher water-transferred intensities than the low-pH protein. In particular, the TM1B and TM3B helices in subunit B are much more water accessible at high $\mathrm{pH}$ than at low $\mathrm{pH}$. Moreover, the extent of hydration is more comparable between the two subunits at high $\mathrm{pH}$ compared to the low-pH complex. This increased similarity of the hydration extent at high $\mathrm{pH}$ is exemplified by the G26 pair, and is consistent with the smaller chemical shift asymmetry of the protein at high $\mathrm{pH}$. We attribute these observations to the more parallel orientations of the TM helices in the high-pH complex, which reduce the difference in the degree of opening between the two ends of the helical bundle. In comparison, the low-pH EmrE-TPP complex shows much 
Table 1 NMR and refinement statistics for $F_{4}-$ TPP + bound S64V-EmrE structure in DMPC bilayers at pH 8.0.

Monomer A

NMR distance and dihedral constraints

Dipolar coupling measurements

Distance constraints

Total dihedral-angle restraints

$\phi$

$\psi$

$\chi_{1}$

Structure statistics

Violations (mean \pm s.d.)

Distance constraints $(\AA)$

Max. distance-constraint violation $(\AA)$

$\phi$ Dihedral-angle constraints $\left(^{\circ}\right)$

$\psi$ Dihedral-angle constraints $\left(^{\circ}\right)$

Max. $\phi$ dihedral-angle violation $\left(^{\circ}\right)$

Max. $\psi$ dihedral-angle violation $\left(^{\circ}\right)$

Average pairwise r.m.s.d $(\AA)^{a}$

Protein heavy atom

Protein backbone

Protein transmembrane heavy atom

Protein transmembrane backbone

Ligand heavy

Ligand center ${ }^{b}$

$\begin{array}{ll}63 & 52 \\ 205 & 182 \\ 83 & \\ 83 & 65 \\ 43 & 65 \\ & 33\end{array}$

$\begin{array}{ll}0.004 \pm 0.042 & 0.017 \pm 0.112 \\ 0.85 & 1.62 \\ 0.302 \pm 1.815 & 0.545 \pm 3.454 \\ 0.744 \pm 4.289 & 0.508 \pm 3.905 \\ 17.2 & 31.9 \\ 53.8 & 60.1 \\ & \\ 3.43 \pm 1.06 & \\ 2.85 \pm 0.95 & \\ 2.66 \pm 0.86 & \\ 2.23 \pm 0.83 & \\ 1.84 \pm 0.61 & \\ 1.30 \pm 0.64 & \end{array}$

a Pairwise RMSD was calculated among 10 lowest-violation structures between the two independent MD runs after the refinement had equilibrated.

bigand center is operationally defined as phosphorus and the four directly bonded carbon atoms of $\mathrm{F}_{4}$-TPP + .

Table 2 Protein-substrate distances extracted from the NMR-refined structural models.

\begin{tabular}{|c|c|c|c|c|}
\hline & \multicolumn{2}{|l|}{ pH 8.0} & \multicolumn{2}{|l|}{ pH 5.8} \\
\hline & Monomer A & Monomer B & Monomer A & Monomer B \\
\hline P-E14 C & $6.6 \pm 0.7 \AA$ & $7.5 \pm 0.5 \AA$ & $5.6 \pm 0.3 \AA$ & $7.5 \pm 1.0 \AA$ \\
\hline $\mathrm{P}-\mathrm{Y} 40 \mathrm{O} \zeta$ & $7.6 \pm 1.8 \AA$ & $11.2 \pm 1.2 \AA$ & $6.8 \pm 0.5 \AA$ & $16.7 \pm 0.3 \AA$ \\
\hline P-Y60 O & $9.0 \pm 1.2 \AA$ & $6.9 \pm 1.1 \AA$ & $9.8 \pm 0.7 \AA$ & $5.9 \pm 0.4 \AA$ \\
\hline$P-W 63 N \varepsilon$ & $5.6 \pm 0.5 \AA$ & $5.7 \pm 0.9 \AA$ & $6.0 \pm 0.4 \AA$ & $5.6 \pm 0.3 \AA$ \\
\hline Min. Fa-E14 C $\delta$ & $4.7 \pm 0.8 \AA$ & $5.2 \pm 0.6 \AA$ & $4.6 \pm 0.5 \AA$ & $6.5 \pm 0.7 \AA$ \\
\hline Min. $F^{a}-Y 40$ OS & $6.0 \pm 1.0 \AA$ & $9.5 \pm 0.8 \AA$ & $6.2 \pm 0.6 \AA$ & $12.2 \pm 0.4 \AA$ \\
\hline 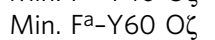 & $6.1 \pm 0.8 \AA$ & $5.7 \pm 0.6 \AA$ & $6.9 \pm 0.4 \AA$ & $5.6 \pm 0.5 \AA$ \\
\hline Min. $F^{a}-W 63 \mathrm{~N} \varepsilon$ & $4.8 \pm 0.3 \AA$ & $4.9 \pm 0.7 \AA$ & $5.8 \pm 0.3 \AA$ & $5.7 \pm 0.4 \AA$ \\
\hline
\end{tabular}

The average distances and standard deviations are from the ensemble of 10 minimum constraint-violating structures in the final $240 \mathrm{~ns}$ of MD trial 1 and $160 \mathrm{~ns}$ of MD trial 2 . P refers to TPP phosphorus atom while protein atom is denoted by standard IUPAC nomenclature.

aDistances of the nearest fluorine to specific protein atoms.

higher hydration for subunit A than subunit B, in good agreement with the larger conformational asymmetry of the two subunits. These spectral observations are borne out by the MD equilibrated structural ensemble, as the structure calculation explicitly solvated the protein-ligand complex in lipid bilayers. Figure 6e shows membrane-embedded water molecules whose oxygen atoms lie within $15 \AA$ of any of the ligand atoms. Strikingly, the ligand-binding site is much more hydrated in the high-pH complex: a total of 69 water molecules are found in the ligandbinding pocket, and these water molecules approach the ligand from both sides of the lipid bilayer. In comparison, only 23 water molecules are found in the ligand-binding pocket in the low-pH complex; moreover, they approach the ligand only from one side, the putative periplasmic side, of the lipid bilayer.

\section{Discussion}

The high-pH structure the EmrE-TPP complex is consistent with the available biochemical data and suggests a mechanism for how proton binding drives release of high-affinity substrates from this promiscuous transporter. Extensive mutagenesis of residues throughout EmrE have demonstrated that E14 in TM1 is critical for binding both drug-like substrates and protons for the transport activity ${ }^{40-42}$. Residues in TM2 and TM3 are important for substrate binding and substrate specificity ${ }^{4,43-45}$. TM3 also contains a putative hinge region that is important for controlling the rate of alternating access of the transporter. TM4 is outside the binding pocket and contains the dimerization motif that stabilizes this highly dynamic homodimer $24,32,46$. The measured substrateprotein distances shown here and in the previous study are the shortest for residues in TM1-3 at both low and high $\mathrm{pH}$, consistent with the substrate-binding site inferred from mutagenesis and the overall structural organization of the transporter observed in low-resolution $\mathrm{EM}^{9}$ and crystal structures ${ }^{8}$. The asymmetry of chemical shifts between subunits A and B is the largest in TM3 (Supplementary Fig. 4). This is consistent with the proposal that the hinge and the difference in local conformation of this helix ${ }^{21}$ between the two subunits control the asymmetric structure of the EmrE dimer and the rate of alternating access.

The fact that these significant structural changes result exclusively from a $\mathrm{pH}$ change is remarkable. Deprotonation of the two 



pH 5.8
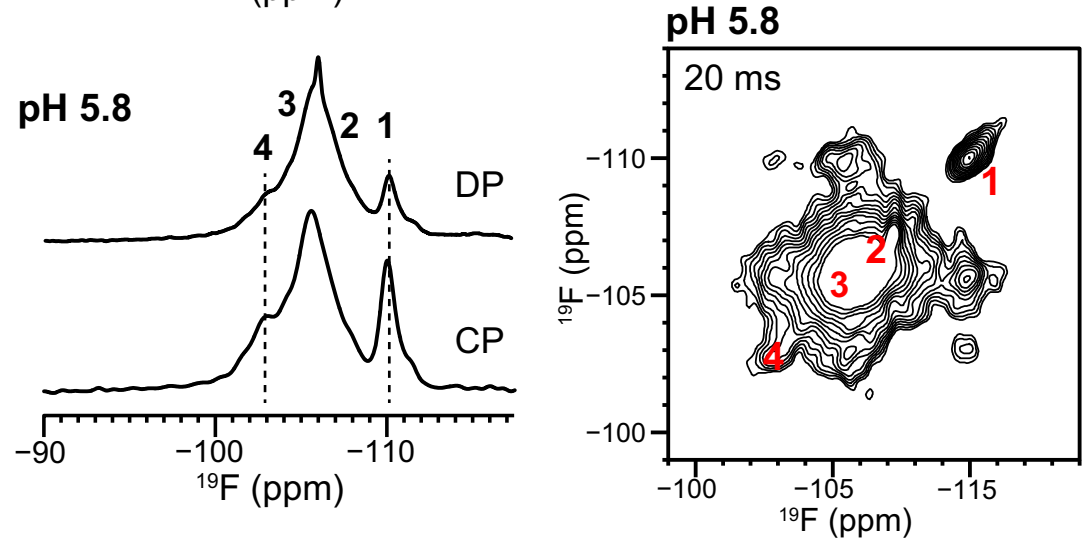



pH 5.8



Fig. $5{ }^{19} \mathbf{F}$ NMR spectra of $\mathbf{F}_{4}-\mathrm{TPP}+$ to probe substrate structure and dynamics. a $1 \mathrm{D}{ }^{19} \mathrm{~F} \mathrm{NMR}$ spectra of $\mathrm{F}_{4}-\mathrm{TPP}+$ bound to $\mathrm{EmrE}$ at $\mathrm{pH} 8.0$ and $\mathrm{pH} 5.8$. Multiple ${ }^{19} \mathrm{~F}$ chemical shifts are resolved, indicating heterogeneous structural environments of the four fluorines. b $2 \mathrm{D}{ }^{19} \mathrm{~F}-{ }^{19} \mathrm{~F}$ correlation spectra of EmrEbound $\mathrm{F}_{4}-\mathrm{TPP}+$ at $\mathrm{pH} 8.0$ (blue) and $\mathrm{pH} 5.8$ (black), measured with a mixing time of $20 \mathrm{~ms} . \mathrm{c}^{19} \mathrm{~F}$ exchange buildup and decay curves for the resolved peak 4 at $\mathrm{pH} 8.0$ and the resolved peak 1 at $\mathrm{pH} 5.8$. Data are presented as mean values $+/-2 \sigma$. Error of intensity values was propagated from spectral signal to noise, while fitting parameter errors were estimated by Monte Carlo methods. These ${ }^{19} \mathrm{~F}$ spectra were measured under $38 \mathrm{kHz} \mathrm{MAS}$ at a sample temperature of $\sim 285 \mathrm{~K}$. Cross-peak intensities are provided as a Source Data file.

\begin{tabular}{|c|c|c|c|}
\hline pH & $\Delta G(\mathrm{~kJ} / \mathrm{mol})$ & $\Delta H(\mathrm{~kJ} / \mathrm{mol})$ & $-T \Delta S(\mathrm{~kJ} / \mathrm{mol})$ \\
\hline 5.5 & $-25.3 \pm 0.3$ & $-40.0 \pm 0.6$ & $15 \pm 0.7$ \\
\hline 6.5 & $-34.9 \pm 0.4$ & $-31.5 \pm 1.7$ & $-3.4 \pm 1.7$ \\
\hline 7.5 & $-41.1 \pm 0.8$ & $-19.1 \pm 0.6$ & $-21.0 \pm 1.0$ \\
\hline 8.5 & $-46.0 \pm 0.6$ & $-20.7 \pm 2.7$ & $-25.3 \pm 2.8$ \\
\hline
\end{tabular}

Original data for TPP+ binding to WT EmrE was reported in ${ }^{18}$. Here we present the average $\mathrm{pH}-$ dependent binding affinity, enthalpy, and entropy for substrate-protein interaction, independent of any contribution from buffer ionization.

E14 residues not only symmetrizes the $\mathrm{TPP}^{+}$position but also increases the separation between the two TM1 helices. The E14 $\mathrm{C} \delta$ - $\mathrm{C} \delta$ distance increased from $11.8 \pm 1.3 \AA$ at low $\mathrm{pH}$ to $13.3 \pm 0.8 \AA$ at high $\mathrm{pH}$. This subtle increase of the E14-E14 separation, and the ensuing change in the binding pocket geometry (Supplementary Fig. 7), translates to a noticeable effect on the drug dynamics, speeding up ligand reorientation rates two-fold compared to the low-pH complex. The motion is likely tetrahedral jumps. $2 \mathrm{D}{ }^{19} \mathrm{~F}-{ }^{19} \mathrm{~F}$ exchange data suggest that this tetrahedral jump is incomplete in geometry: while peak 4 displays equilibrated exchange intensities of $\sim 0.25$ by $80 \mathrm{~ms}$, the other three sites do not fully equilibrate, suggesting that these fluorines are impeded by the protein sidechains. The substrate motion is more complete in the high-pH complex than in the low-pH complex, whose ${ }^{19} \mathrm{~F}$ exchange intensities are less equilibrated (Supplementary Fig. 8d). The differences between the structures and drug dynamics of the low- and high-pH states indicate that ligand dynamics depend both on the size of the binding pocket and on sidechain obstructions.

Comparison of the structures of the EmrE-TPP complex at low and high $\mathrm{pH}$ is most informative in understanding how protonation of one $\mathrm{E} 14$ residue can drive release of the $\mathrm{TPP}^{+}$substrate. It is well established that the apparent affinity of EmrE for druglike substrate is weaker at low $\mathrm{pH}$ than at high $\mathrm{pH}^{42}$, and this is due to a faster substrate off-rate at low $\mathrm{pH}$. This $\mathrm{pH}$-dependent substrate affinity was originally attributed to a simple competition between $\mathrm{TPP}^{+}$and proton for binding to E14. However, we now know that EmrE can bind proton and $\mathrm{TPP}^{+}$simultaneously at low $\mathrm{pH}^{17,39}$, and the two E14 residues are sufficiently far apart from each other that electrostatic coupling is minimal and proton binding and release occur relatively independently of either residue $^{30}$. Examining the structural changes of the EmrE-TPP complex from high $\mathrm{pH}$ to low $\mathrm{pH}$ immediately suggests why $\mathrm{TPP}^{+}$affinity is lower in the protonated state. At high $\mathrm{pH}, \mathrm{TPP}^{+}$ is buried deep within the helical bundle, positioned nearly symmetrically between the two TM1 helices within an elongated binding cavity (Fig. 4 and Supplementary Fig. 7). In contrast, at low $\mathrm{pH}$, the transporter structure is more asymmetric and clearly closed on one side and open on the other. $\mathrm{TPP}^{+}$is positioned much closer to the open end of the binding cavity, primed for release. The weakening of substrate affinity upon proton binding is likely important for speeding up the release of tight-binding substrates so that this promiscuous transporter can rapidly efflux 
a

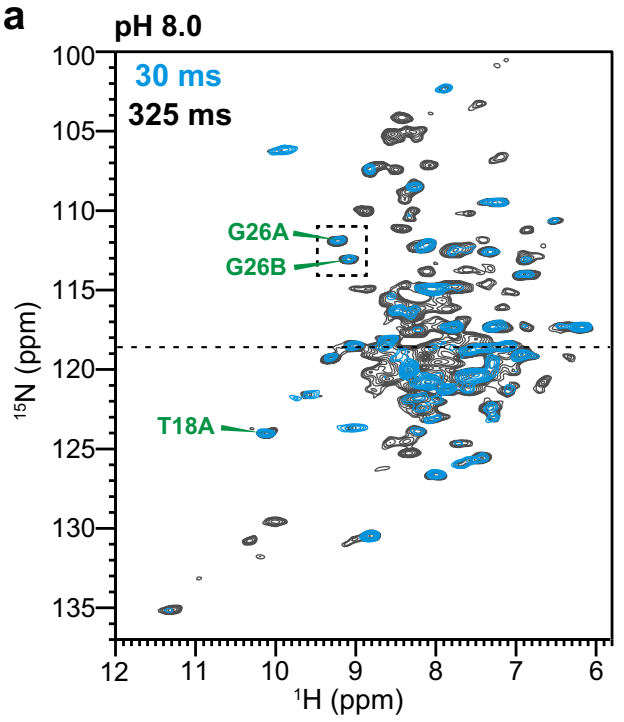

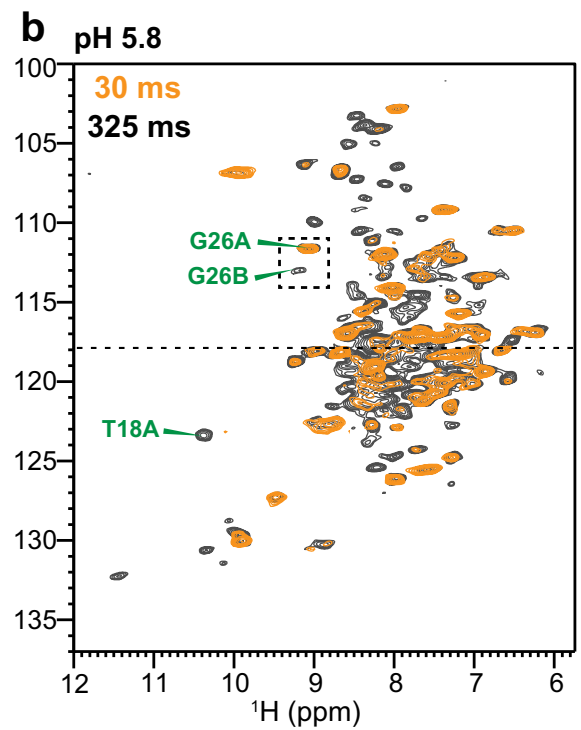
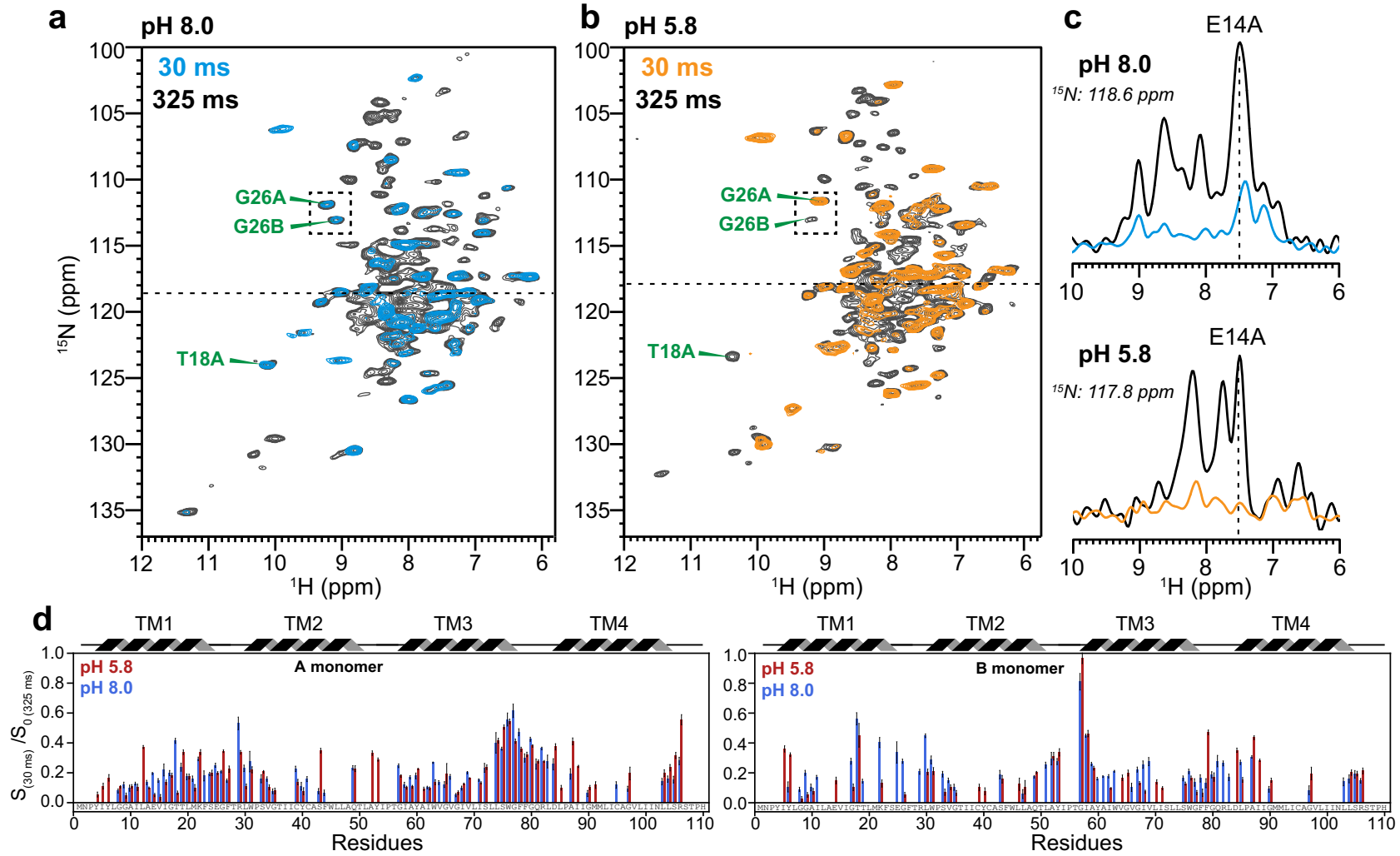

e

pH 8.0

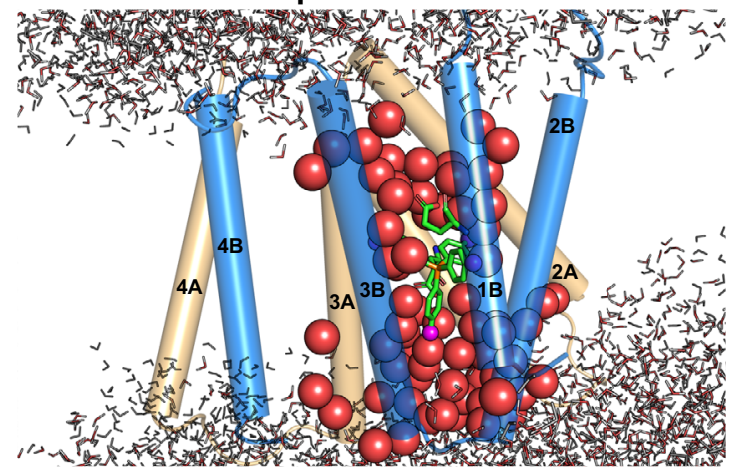

pH 5.8



Fig. 6 Water-edited 2D hNH spectra of TPP ${ }^{+}$-bound EmrE. a pH 8.0 spectra. b pH 5.8 spectra. c $1 \mathrm{D}^{15} \mathrm{~N}$ cross-sections of E14A extracted from the wateredited 2D hNH spectra. The $\mathrm{pH} 8.0$ sample shows higher water-transferred intensities than the $\mathrm{pH} 5.8$ sample. $\mathbf{d}$ Intensity ratios $\mathrm{S} / \mathrm{S}_{0}$ between the 30 and $325 \mathrm{~ms}$ spectra of the water-edited 2D spectra to compare the water accessibilities between the high and low pH proteins. The intensities have been corrected for ${ }^{1} \mathrm{H} \mathrm{R}_{1}$ relaxation. e MD equilibrated membrane-bound structures of the EmrE-TPP ${ }^{+}$complex at pH 8.0 and pH 5.8. Water oxygens within $15 \AA$ of any of the ligand atoms and that are within the lipid bilayer are shown as red spheres. Membrane-surface water molecules are shown as thin lines, and water molecules $>15 \AA$ away from the protein are shown in gray. The number of water molecules is 69 in the high-pH complex but only 23 in the low-pH complex, consistent with the higher water-transferred intensities of the protein at high $\mathrm{pH}$. Moreover, water hydrates the ligand from both sides of the membrane in the high-pH complex, but only accesses the ligand from the periplasmic side in the low-pH complex. The water-edited intensities are provided as a Source Data file.

a broad range of substrates with widely varying affinities ${ }^{17,19}$. The $\mathrm{pH}$-dependent thermodynamic parameters (Table 3) show that higher-affinity drug binding by this promiscuous transporter is driven by increasingly favorable binding entropy. This is consistent with the enhanced ligand dynamics and hydration observed here in the high-pH structure. There is not sufficient ITC data or in vitro transport data available to assess whether the relative promiscuity of EmrE changes appreciably with $\mathrm{pH}$. That remains an open question for further study.

The native environment for EmrE is the inner membrane of $E$. coli. This is an asymmetric environment unlike the in vitro conditions used for structure determination. Usually, the periplasmic $\mathrm{pH}$ is lower than the cytoplasmic $\mathrm{pH}^{47}$. Given the significant structural changes of the protein we determined here as a function of E14 protonation state in a symmetric membrane, it is reasonable to assume that the protonation state of E14 regulates the overall structural change of the protein. We can then infer what the structure might look like in the presence of a transmembrane $\mathrm{pH}$ gradient. Cytoplasmic $\mathrm{pH}$ in E. coli is generally 7.4-7.84, above the $\mathrm{pK}_{a}$ of E14 in the $\mathrm{F}_{4}$ - $\mathrm{TPP}^{+}$-bound transporter. Thus, the open-in conformation of EmrE is expected to have predominantly deprotonated E14 residues and more closely resemble the high-pH structure (Fig. 4a, c, e). In contrast, the periplasmic $\mathrm{pH}$ closely correlates with the $\mathrm{pH}$ of the external 
environment ${ }^{47}$. Thus, when $E$. coli are in an acidic environment like the human gut, the periplasmic $\mathrm{pH}$ will be well below the $\mathrm{pK}_{a}$ of E14 and the structure of EmrE should more closely resemble the low $\mathrm{pH}$ structure (Fig. $4 \mathrm{~b}, \mathrm{~d}, \mathrm{f}$ ).

It is interesting to note that the high-pH structure of the EmrETPP complex is not fully closed on either side of the membrane. This is borne out by the water-accessibility data (Fig. 6a-d) and the MD simulations (Fig. 6e), which show larger water accessibility of the ligand-binding cavity at high $\mathrm{pH}$ than at low $\mathrm{pH}$. Moreover, water molecules approach the ligand from both sides of the membrane at high $\mathrm{pH}$, but only access the ligand from one side of the membrane at low $\mathrm{pH}$. The dual-open conformation at high $\mathrm{pH}$ suggests that after a toxic substrate has bound to the protein from the cytoplasm and EmrE adopts a conformation similar to Supplementary Fig. 7a, there is sufficient space for protons to enter and bind E14 from the periplasmic side. This could then trigger a conformational change to a state similar to the low-pH structure, with the transporter open to the periplasmic side of the membrane. In this outward-facing state, the substrate is bound peripherally to the central transport pore, and is thus primed for release in accord with the enhanced off rate in the drug- and proton-bound state.

\section{Methods}

S64V-EmrE expression and purification. S64V-EmrE was expressed and purified as previously described ${ }^{7}$ using the same procedure as for WT EmrE ${ }^{49} .{ }^{2} \mathrm{H},{ }^{13} \mathrm{C},{ }^{15} \mathrm{~N}$ (CDN)-labeled S64V-EmrE was expressed in ${ }^{2} \mathrm{H}_{2} \mathrm{O}$ media containing $2.5 \mathrm{~g} / \mathrm{L}$ $\mathrm{U}^{2}{ }^{2} \mathrm{H},{ }^{13} \mathrm{C}$ glucose, $1 \mathrm{~g} / \mathrm{L}{ }^{15} \mathrm{NH}_{4} \mathrm{Cl}$, and $0.5 \mathrm{~g} / \mathrm{L}{ }^{2} \mathrm{H},{ }^{13} \mathrm{C},{ }^{15} \mathrm{~N}$-labled ISOGRO. Lysis and purification were performed as previously reported ${ }^{50,51}$ using Ni-NTA affinity column followed by thrombin cleavage of the His-tag and size exclusion chromatography on a S200 column in buffer containing $50 \mathrm{mM}$ MES, $20 \mathrm{mM} \mathrm{NaCl}$, $10 \mathrm{mM}$ decyl-maltoside, $5 \mathrm{mM}$ BME, $\mathrm{pH}$ 7.0.

Solution NMR spectroscopy and $\mathbf{p K}_{\mathrm{a}}$ analysis. Solution NMR data were collected on samples with $1.0 \mathrm{mM}{ }^{2} \mathrm{H},{ }^{15} \mathrm{~N}$ S64V-EmrE in DMPC/DHPC bicelles $(q=0.33$, with a protein to DMPC molar ratio of 1:50). The buffer contained $20 \mathrm{mM} \mathrm{NaCl}, 50 \mathrm{mM}$ sodium acetate, $50 \mathrm{mM}$ MOPS, $50 \mathrm{mM}$ MES, $50 \mathrm{mM}$ bicine, $2 \mathrm{mM}$ TCEP, $0.01 \%$ DSS, and $10 \% \mathrm{D}_{2} \mathrm{O}$. About $10 \mathrm{mg} \mathrm{F}_{4}$-TPP ${ }^{+}$was added to this protein bicelle solution and incubated at $45^{\circ} \mathrm{C}$ overnight to saturate binding, then the excess drug was removed through microcentrifugation. Spectra were measured at $45^{\circ} \mathrm{C}$ on a Bruker $750 \mathrm{MHz}$ spectrometer (Avance) equipped with a TCI cryoprobe. Spectra from 4 different $\mathrm{pH}$ conditions were processed and analyzed using NMRPipe ${ }^{52}$ and CcpNmr Analysis ${ }^{53}$. Chemical shift changes for ${ }^{1} \mathrm{H}$ and ${ }^{15} \mathrm{~N}$ were separately globally fit to a single $\mathrm{pK}_{a}$ value using the following equation (Eq. 1):

$$
\delta=\frac{\delta_{H} 10^{-\mathrm{pH}}+\delta_{D} 10^{-\mathrm{pK}}}{10^{-\mathrm{pH}}+10^{-\mathrm{pK}_{a}}}
$$

where $\delta_{\mathrm{H}}$ and $\delta_{\mathrm{D}}$ are the chemical shifts of the protonated and deprotonated states of each residue, respectively. Six residues in close proximity to E14 with large chemical shift changes with $\mathrm{pH}$ were analyzed. For a single $\mathrm{pK}_{a}$, the modified Henserson-Hasselbach equation describes the chemical shift for each residue $(\delta)$ as a function of $\mathrm{pH}^{15,54,55}$.

Reconstitution and preparation of solid-state NMR samples. CDN-labeled S64V-EmrE was reconstituted into $d_{54}$-DMPC (Avanti Polar Lipids) liposomes at a protein monomer to lipid molar ratio (P: L) of 1: 25. DMPC was resuspended in $50 \mathrm{mM}$ MES, $20 \mathrm{mM} \mathrm{NaCl}, \mathrm{pH} 8.0$ buffer at $20 \mathrm{mg} / \mathrm{mL}$. The lipid mixture was incubated at $45^{\circ} \mathrm{C}$ for $1 \mathrm{~h}$ to hydrate, then bath-sonicated for 1 min before addition of $0.5 \%$ octyl-glucoside $(\mathrm{OG})$ followed by $30 \mathrm{~s}$ bath sonication. The lipid mixture was incubated at $45^{\circ} \mathrm{C}$ for an additional $15 \mathrm{~min}$ before mixing with purified S64VEmrE solution. After $20 \mathrm{~min}$ incubation at room temperature (RT), Amberlite (Supelco) was added $(3 \times 30 \mathrm{mg}$ Amberlite per $\mathrm{mg}$ total detergent) to remove the detergent. The amberlite was removed after $16-24 \mathrm{~h}$ by simple filtration. Liposomes were collected by ultracentrifugation $\left(165,000 \times g, 6^{\circ} \mathrm{C}, 2 \mathrm{~h}\right)$ and resuspended in a small volume $(\sim 20 \mathrm{mg} / \mathrm{mL}$ lipid concentration) of buffer. To ensure complete detergent removal, the sample was dialyzed against $1 \mathrm{~L}$ of the same buffer $(50 \mathrm{mM}$ MES, $20 \mathrm{mM} \mathrm{NaCl}, \mathrm{pH} 8.0$ ) with buffer change every $24 \mathrm{~h}$ over a $72 \mathrm{~h}$ period. The sample was then incubated with excess solid $\mathrm{F}_{4}-\mathrm{TPP}^{+}$at RT with end-over-end rocking for at least $16 \mathrm{~h}$. Excess $\mathrm{F}_{4}-\mathrm{TPP}^{+}$was removed using microcentrifugation $(7,500 \times g, 5 \mathrm{~min})$. Proteoliposomes were then pelleted at $100,000 \times g, 4^{\circ} \mathrm{C}, 2 \mathrm{~h}$ in an ultracentrifuge. Proteoliposomes were dried to $\sim 40 \%$ hydration by mass in a desiccator. Samples were centrifuged into 1.9 and $1.3 \mathrm{~mm}$ MAS rotors. Two rotors were packed: a $1.9 \mathrm{~mm}$ sample of $\mathrm{CDN}-\mathrm{EmrE}$ sample containing $\sim 3.5 \mathrm{mg}$ protein in $\sim 15 \mathrm{mg}$ proteoliposomes, and a $1.3 \mathrm{~mm}$ sample of CDN-EmrE containing $\sim 0.9 \mathrm{mg}$ protein in $\sim 3.6 \mathrm{mg}$ proteoliposomes.

Solid-state NMR experiments. All MAS SSNMR experiments were conducted on a $600 \mathrm{MHz}$ Bruker AVANCE II spectrometer. Chemical shift assignment and protein hydration experiments were carried out under $55 \mathrm{kHz}$ MAS on a $1.3 \mathrm{~mm}$ $\mathrm{HCN}$ probe, whereas $2 \mathrm{D}^{1} \mathrm{H}_{-}{ }^{15} \mathrm{~N}$ resolved ${ }^{1} \mathrm{H}-{ }^{19} \mathrm{~F}$ REDOR experiments and $2 \mathrm{D}$ ${ }^{19} \mathrm{~F}-1{ }^{19} \mathrm{~F}$ exchange experiments were conducted under $38 \mathrm{kHz}$ MAS on a $1.9 \mathrm{~mm}$ HFX probe. Sample temperature was controlled by matching the chemical shift of water in the proteoliposomes between samples and probes. The effective sample temperatures were estimated from the water ${ }^{1} \mathrm{H}$ chemical shifts using the equation $T_{\text {eff }}(\mathrm{K})=96.9\left(7.83-\delta_{\mathrm{H} 2 \mathrm{O}}\right)$ where $\delta_{\mathrm{H} 2 \mathrm{O}}$ is the observed water chemical shift ${ }^{56}$. By keeping the water ${ }^{1} \mathrm{H}$ chemical shift at $4.89 \mathrm{ppm}$ using appropriate bearing temperatures, we maintained a constant sample temperature of $285 \mathrm{~K}$ for all experiments. Detailed experimental conditions are provided in Supplementary Table 6.

Eight ${ }^{1} \mathrm{H}$-detected 3D MAS correlation experiments were used to assign the chemical shifts of $\mathrm{pH} 8.0$ EmrE. Supplementary Fig. 11 shows the pulse sequences of those experiments that were not included in our low-pH EmrE study ${ }^{39}$ and the pulse sequences of the $2 \mathrm{D}$ water-edited experiments. The hCANH, hCOcaNH, and hcaCBcaNH experiments allow intra-residue CA, CO, and CB assignment, while the $\mathrm{hCAcoNH}, \mathrm{hCONH}$, and hcaCBcacoNH experiments allow sequential residue assignment. In addition, amide-to-amide 3D correlation was achieved using the HncacoNH (Supplementary Fig. 11c) and hNcacoNH (Supplementary Fig. 11d) experiments, which allow inter-residue $\mathrm{H}_{i-1}-\mathrm{N}_{\mathrm{i}} \mathrm{H}_{\mathrm{i}}$ and $\mathrm{N}_{i-1}-\mathrm{N}_{\mathrm{i}} \mathrm{H}_{\mathrm{i}}$ assignment, respectively, to further disambiguate the backbone walk ${ }^{57}$. The coherence transfer steps and resonance assignment connectivities of these eight ${ }^{1} \mathrm{H}$-detected experiments are summarized in Supplementary Table $1 .{ }^{15} \mathrm{~N}-{ }^{13} \mathrm{C}$ correlation experiments used specific $\mathrm{CP}$ for polarization transfer ${ }^{58}$. Under $55 \mathrm{kHz}$ MAS, the double-quantum (DQ) matching condition of $30 \mathrm{kHz}{ }^{13} \mathrm{C}$ and $25 \mathrm{kHz}{ }^{15} \mathrm{~N}$, or $35 \mathrm{kHz}{ }^{15} \mathrm{~N}$ and $20 \mathrm{kHz}{ }^{13} \mathrm{C}$, was used to achieve selective ${ }^{15} \mathrm{~N}-{ }^{13} \mathrm{C}$ polarization transfer. ${ }^{13} \mathrm{CA}-{ }^{13} \mathrm{CO}$ polarization transfer was achieved using the DQ DREAM sequence under $55 \mathrm{kHz}$ MAS. Due to off-resonance effects, a short 1.3-1.6 $\mu$ s trim pulse (marked as a $\theta$ pulse) was used after the spin lock to rotate the magnetization to the $X Y$ plane ${ }^{59}$. For the two CB-NH correlation experiments (Fig. S11a, b), outand-back CA-CB-CA INEPT transfer was used, using the Q3 shaped pulse for $180^{\circ}$ pulses to invert aliphatic coherences while not inverting CO. A $6.5 \mathrm{~ms}$ INEPT mixing period was used for both the creation and reconversion of the antiphase magnetization, for a total of $13 \mathrm{~ms}$ of transfer time. Low-power ${ }^{1} \mathrm{H}$ decoupling was employed for all SSNMR experiments with either CW irradiation or the WALTZ16 scheme $e^{60}$. These ${ }^{1} \mathrm{H}$-detected MAS NMR experiments employed MISSISPPI to suppress the water signal, using $150-200 \mathrm{~ms}$ of $15 \mathrm{kHz}$ irradiation ${ }^{61}$.

Water-edited NMR experiments were carried out with $2 \mathrm{D} \mathrm{hNH}$ detection by inserting a water-selective echo and water-to-protein ${ }^{1} \mathrm{H}$ spin diffusion mixing period following proton excitation and before CP to ${ }^{15} \mathrm{~N}$ (Supplementary Fig. 11e). A $3.5 \mathrm{~ms}$ Gaussian pulse with $5 \%$ truncation and 400 points for the shape was used to selectively refocus water coherence within a $3.6 \mathrm{~ms}$ echo, during which all protein coherence is destroyed by $T_{2}$ relaxation. Due to fast MAS suppressing spin diffusion, long mixing times of $325 \mathrm{~ms}$ for the equilibrated $S_{0}$ spectrum and $30 \mathrm{~ms}$ for the edited $S$ spectra were needed, between which significant $T_{1}$ relaxation occurs. To account for relaxation between the edited and equilibrated spectra, site-resolved $T_{1}$ measurements were carried out through saturation recovery using the pulse sequence in

Supplementary Fig. 11f. After the pre-scan delay d1, we inserted a MISSISSIPPI dephasing block to saturate all ${ }^{1} \mathrm{H}$ magnetization in the sample. Following this saturation, a variable delay allows $\mathrm{T}_{1}$ relaxation to occur before ${ }^{1} \mathrm{H}$ excitation. The experiments were run in a constant-time fashion, where the combined prescan delay and $\tau_{\text {relax }}$ were set to 3,4 , or $5 \mathrm{~s}$ depending on the relaxation time $\tau_{\text {relax }}$ used. ${ }^{1} \mathrm{H}-{ }^{19} \mathrm{~F}$ REDOR measurements and $2 \mathrm{D}{ }^{19} \mathrm{~F}-{ }^{19} \mathrm{~F}$ exchange experiments were run as described previously ${ }^{39}$ to allow for direct comparison of the data between the two samples of distinct $\mathrm{pH}$.

${ }^{19} \mathrm{~F}$ chemical shifts were externally referenced to the $-122.1 \mathrm{ppm}$ signal of $5 \mathrm{~F}$ tryptophan on the $\mathrm{CF}_{3} \mathrm{Cl}$ scale ${ }^{62} .{ }^{13} \mathrm{C}$, and ${ }^{15} \mathrm{~N}$ chemical shifts were internally referenced to the DSS-referenced chemical shifts of the solution-state values. The G90A site was previously shown to have little chemical shift perturbation with $\mathrm{pH}^{15}$, and was therefore chosen as the referencing site; the ${ }^{1} \mathrm{H}$ and ${ }^{15} \mathrm{~N}$ values were set to $8.5 \mathrm{ppm}$ and $107.4 \mathrm{ppm}$, respectively, and the ${ }^{13} \mathrm{C}$ reference was set from the hCANH peak for this residue at $47.6 \mathrm{ppm}$.

Chemical shift assignment. The SSNMR spectra were processed in the Bruker Topspin 3.5 software package with zero-filling, apodization, and Fouriertransformation (FT). Spectra acquired in blocks for signal averaging were added in the time domain prior to FT using a custom Python script that utilized the NMRGlue and NumPy Python packages ${ }^{63,64}$. Chemical shift assignment and 3D spectral plotting were performed in NMRFAM-Sparky ${ }^{65} .1 \mathrm{D}$ and $2 \mathrm{D}$ correlation spectra were plotted using Bruker Topspin's XWINPLOT. Comparisons of chemical shifts between protein monomers and samples were computed in Python and plotted with Matplotlib ${ }^{66}$. The asymmetry of protein monomer chemical shifts was 
calculated using composite $\mathrm{H}^{\mathrm{N}}$ and $\mathrm{N}^{\mathrm{H}}$ chemical shifts according to Eq. 2:

$$
\Delta \omega_{\mathrm{NH}}=\sqrt{\frac{1}{2}\left[\Delta \omega_{\mathrm{H}}^{2}+\left(0.10 * \Delta \omega_{\mathrm{N}}\right)^{2}\right]}
$$

Here $\Delta \omega_{\mathrm{H} / \mathrm{N}}$ is the ${ }^{1} \mathrm{H}$ and ${ }^{15} \mathrm{~N}$ chemical shift difference between monomers $\mathrm{A}$ and $\mathrm{B}$. The composite $\mathrm{CA}$ and $\mathrm{CO}$ chemical shift difference was calculated similarly but without the factor of 0.10 for the gyromagnetic ratio difference. Protein $\phi, \psi$, and $\chi_{1}$ torsion angles were predicted using the TALOS-N software ${ }^{67}$ based on the measured non-H chemical shifts. A deuterium isotope correction was applied to the $\mathrm{C} \alpha$ and $\mathrm{C} \beta$ chemical shifts.

2D ${ }^{19}$ F-19F exchange analysis. We quantified the exchange rates between four peaks in the $2 \mathrm{D}{ }^{19} \mathrm{~F}-{ }^{19} \mathrm{~F}$ correlation spectra at both high and low $\mathrm{pH}$. Peak volumes were integrated in Topspin using the same peak areas across all mixing times, and were normalized with respect to the sum of the integrated intensities of all peaks in each row. Thus, at short mixing times where most intensities reside on the diagonal, the three cross peaks should have normalized intensities near 0 , while at sufficiently long mixing times, the diagonal and three cross peaks should equilibrate to similar intensities of $\sim 0.25$. In practice, due to spectral overlap, the measured intensities deviate from the ideal equilibrium values. The normalized intensities were fit to a single-exponential decay (Eq. 3) and single-exponential buildup (Eq. 4) equations for the diagonal and cross peaks, respectively:

$$
\begin{gathered}
I_{\text {Diagonal }}\left(t_{\text {mix }} \mid Y_{0}, P, k\right)=\left(Y_{0}-P\right) * \mathrm{e}^{-k * t_{\text {mix }}}+P \\
I_{\text {CrossPeak }}\left(t_{\text {mix }} \mid Y_{0}, P, k\right)=\left(P-Y_{0}\right) *\left(1-\mathrm{e}^{-k * t_{\text {mix }}}\right)+Y_{0}
\end{gathered}
$$

Here $Y_{0}$ is the initial intensity, $P$ is the plateau value, and $k$ is the exponential buildup or decay rate, and $t_{\mathrm{mix}}$ is the mixing time. Fitting was performed in the SciPy optimization module of python ${ }^{68}$. Errors are $2 \sigma$ for individual points, and were estimated from the signal-to-noise ratios (SNR) of the spectral peaks. The SNR for a reference peak was measured using Sparky's built-in routine using 1000 random points to calculate SNR, and the SNR for the remaining peaks was estimated from this value by scaling by the intensity ratio of the reference peak to the remaining peaks. The errors were propagated using Eq. $5^{36}$.

$$
\epsilon_{i}=2 * \sigma=2 * I_{i} * \sqrt{\left(\frac{1}{\mathrm{SNR}_{i}}\right)^{2}+\left(\frac{1}{\mathrm{SNR}_{\text {norm }}}\right)^{2}}
$$

where $I_{\mathrm{i}}$ is the normalized intensity of the $i$-th peak, $\mathrm{SNR}_{\mathrm{i}}$ is the SNR of the $i$-th peak, and $\mathrm{SNR}_{\text {norm }}$ is the relative SNR of the normalization factor for the row. The errors for the fitting parameters were determined using Monte Carlo analysis as shown before for $T_{1 \rho}$ experiments ${ }^{69}$. Briefly, we simulated 1000 additional datasets for each decay and buildup curve by adding a random value from a Gaussian distribution centered with $\mu=0, \sigma=0.3$ (chosen to give values mostly within \pm 1 ) multiplied by the error for each point, $\varepsilon_{\mathrm{I}}$. In this way, we created 1000 new datasets with the points moving randomly within the error bar for each point, but with a Gaussian distribution around the actual measured value. These 1000 datasets were then fit to the same buildup or decay curves to obtain the fitting parameters for each simulated dataset. The standard deviation of the fit parameters in the Monte Carlo datasets was then used as the fitting parameter error reported.

${ }^{1} \mathbf{H}-{ }^{19} \mathbf{F}$ distance extraction. $2 \mathrm{D}$ hNH-resolved $\mathrm{H}^{\mathrm{N}}-{ }^{19} \mathrm{~F}$ REDOR distance restraints were determined as described before ${ }^{36,37,39}$. Briefly, we integrated peak volumes in the $2 \mathrm{D}{ }^{1} \mathrm{H}-{ }^{19} \mathrm{~F}$ REDOR-edited $\mathrm{hNH} S_{0}$ and $S$ spectra to obtain the intensity ratios $S / S_{0}$ for all mixing times for each protein $\mathrm{H}^{\mathrm{N}}$. Using the SIMPSON software package, we simulated the two-spin REDOR dephasing curves for distances of 3.0-15.0 $\AA$ in $0.1 \AA$ increments, including the magnitude and asymmetry parameters of the ${ }^{19} \mathrm{~F}$ CSA, but with default orientation ${ }^{70}$. Finite-pulse effects were explicitly included in the SIMPSON program. RF inhomogeneity was accounted for by simulating for pulse flip angles of $180^{\circ}-145^{\circ}$ in $5^{\circ}$ increments, weighted by a half-Gaussian function centered at $180^{\circ}$ and a standard deviation of $15^{\circ 36,37}$. The REPULSION320 scheme with 32 gamma angles was used for powder averaging ${ }^{71}$. The best-fit ${ }^{1} \mathrm{H}-{ }^{19} \mathrm{~F}$ distance was extracted by minimizing the RMSD between the simulated and measured $S / S_{0}$ values. The uncertainty in the best-fit distance was set by the same RMSD threshold of 0.2 as the previous study ${ }^{39}$. Distances below this RMSD value were considered significant. In cases where little to no dephasing was observed, we set the distance upper uncertainty to $40 \AA$, the approximate longest possible distance in the dimer (Tables S2, S4). For residues whose signals overlap in the $2 \mathrm{D} \mathrm{hNH}$ spectrum, the lower-limit distance uncertainty was increased.

Analysis of water-edited spectra under fast MAS. Protein hydration was investigated using a water-edited $2 \mathrm{D}$ experiment where water ${ }^{1} \mathrm{H}$ polarization was selectively excited and transferred to the protein and detected in $2 \mathrm{D} \mathrm{hNH}$ spectra (Supplementary Fig. 10e). The hydration intensities were analyzed using a modified procedure from the previously reported approach ${ }^{72-74}$ to account for the effects of fast MAS. Because fast MAS suppresses spin diffusion, it was necessary to use longer ${ }^{1} \mathrm{H}$ mixing times for both the equilibrated $S_{0}$ spectrum $(325 \mathrm{~ms})$ and the edited $S$ spectrum $(30 \mathrm{~ms})$. The edited spectra were signal averaged with $3.5-4.5$ times as many scans as the equilibrated spectra to obtain sufficient SNR. Siteresolved hydration intensities were calculated by dividing the integrated peak volumes of the $30 \mathrm{~ms} S$ spectrum by the corresponding peak volumes in the $325 \mathrm{~ms}$ $S_{0}$ spectrum. However, at $325 \mathrm{~ms}$ mixing, ${ }^{1} \mathrm{H} \mathrm{T} \mathrm{T}_{1}$ relaxation is non-negligible, causing the $S / S_{0}$ values to be larger than 1 for some residues. To correct for this $\mathrm{T}_{1}$ relaxation, we measured $2 \mathrm{D} \mathrm{hNH}$ resolved ${ }^{1} \mathrm{H}$ saturation-recovery spectra (Supplementary Fig. 10) to obtain site-specific ${ }^{1} \mathrm{H} \mathrm{R}_{1}$ rates at both $\mathrm{pH}$ values. The ${ }^{1} \mathrm{H} \mathrm{R}_{1}$ rates were extracted from the integrated peak volumes using the same integration areas as for the water-edited spectra. The intensities of each $\mathrm{H}^{\mathrm{N}}$ site were normalized to the maximum intensity for that site. Error bars for each point were propagated from the SNR of the spectra according to Eq. 6 :

$$
\epsilon_{i}=2 * \sigma=2 * I_{i} * \sqrt{\left(\frac{1}{\mathrm{SNR}_{i}}\right)^{2}+\left(\frac{1}{\mathrm{SNR}_{\text {norm }}}\right)^{2}}
$$

The saturation recovery curves were fit to a single-exponential buildup function (Eq. 7) to obtain the $R_{1}$ values:

$$
\mathrm{I}\left(t_{\text {relax }} \mid P, R_{1}\right)=P *\left(1-\mathrm{e}^{-R_{1} * t_{\text {relax }}}\right)
$$

The $R_{1}$ uncertainty, $\sigma_{R 1}$, was estimated using the same Monte Carlo method described above for the ${ }^{19} \mathrm{~F}$ exchange analysis. With the site-specific $R_{1}$ rates known, the hydration $S / S_{0}$ values were corrected for relaxation between the two mixing times (325 and $30 \mathrm{~ms}$ ) according to Eq. 8:

$$
\mathrm{H}\left(\frac{S}{S_{0}}, R_{1}\right)=\frac{S}{S_{0} * \mathrm{e}^{R_{1}\left(t_{2}-t_{1}\right)}}=\frac{S}{S_{0}} * \mathrm{e}^{R_{1}\left(t_{1}-t_{2}\right)}
$$

The error of the corrected $S / S_{0}$ value, $H$, is a result of the errors in the $S, S_{0}$, and $R_{1}$ values and was calculated according to Gauss' error propagation according to Eqs. 9 and 10:

$$
\begin{gathered}
\sigma_{S / S_{0}}=\frac{S}{S_{0}} * \sqrt{\left(\frac{1}{\mathrm{SNR}_{S}}\right)^{2}+\left(\frac{1}{\mathrm{SNR}_{S_{0}}}\right)^{2}} \\
\sigma_{H}=\sqrt{\mathrm{e}^{2 R_{1}\left(t_{1}-t_{2}\right)} * \sigma_{S / S_{0}}^{2}+\left[\frac{S}{S_{0}}\left(t_{1}-t_{2}\right) \mathrm{e}^{R_{1}\left(t_{1}-t_{2}\right)}\right]^{2} * \sigma_{R_{1}}^{2}}
\end{gathered}
$$

Using this method, the water-edited intensities can be compared fairly for different residues with different $R_{1}$ rates and for two $\mathrm{pH}$ conditions.

Structure calculation of EmrE with bound $\mathbf{F}_{\mathbf{4}}-\mathbf{T P P}+$ at high $\mathbf{p H}$. The high-pH EmrE-TPP structure was calculated in a two-stage process similar to that detailed recently ${ }^{39} . \mathrm{F}_{4}$-TPP ${ }^{+}(\mathrm{PDB}$ : VCJ) was first docked into the $\mathrm{pH} 5.8$ protein structure (PDB: 7JK8) using ${ }^{1} \mathrm{H}-{ }^{19} \mathrm{~F}$ distance constraints measured at $\mathrm{pH}$ 8.0. This docking orients the ligand and disambiguates the four-fold degenerate ${ }^{1} \mathrm{H}-{ }^{19} \mathrm{~F}$ constraints. The protein was then subject to all-atom molecular dynamics refinement in explicitly solvated DMPC bilayers. In both stages, the two E14 residues were modeled in the deprotonated state. Docking was performed using the HADDOCK v2.4 webserver 75,76 . The "active residues" list was set to a list of 18 protein residues between the A and B subunits which are known to with the ligand from prior biochemical data ${ }^{77}$ and our REDOR data. The new ${ }^{1} \mathrm{H}-{ }^{19} \mathrm{~F}$ REDOR distances were used as "unambiguous" constraints which are always enforced, with ranges determined by the RMSD cutoff of 0.2 (Supplementary Fig. 5). At this stage, the ${ }^{19} \mathrm{~F}$ atoms were input as four-fold ambiguous. Both ambiguous (active-residue defined) and unambiguous (distance measurement defined) constraints used default HADDOCK energy weighting values of $10.0,10.0,50.0$, and 50.0 for the hot, cool1, $\operatorname{cool} 2$, and $\operatorname{cool} 3$ stages of the docking simulations. Docking was performed in DMSO and started with 1000 structures, from which 200 were outputted after refinement. These 200 structures were analyzed against the four-fold ambiguous ${ }^{1} \mathrm{H}-{ }^{19} \mathrm{~F}$ distance constraints using an integrated Python-Pymol script ${ }^{63,68}$ to select the structures that best agree with the experimental data (Supplementary Table 2). We scored the structures by the lowest sum-total of violations and the least number of violations to create two separate ensembles (Supplementary Fig. 6a and Supplementary Table 3 ). The lowest violation by each criterium was used to structurally disambiguate the ${ }^{1} \mathrm{H}-{ }^{19} \mathrm{~F}$ pairs to create 387 distance constraints from the 116 dipolar coupling measurements ${ }^{39}$ (Supplementary Table 4). The majority (92) of the measured dipolar couplings are weak and correspond to long distances that are four-fold degenerate. About 18 dipolar couplings are strong and can be assigned to unambiguous $\mathrm{H}^{\mathrm{N}}-\mathrm{F}$ pairs based on docking. Each of the two lowest violation structures was used for further refinement by MD simulations in GROMACS.

The docked high-pH EmrE-TPP complexes were aligned to the membrane normal using the OPM webserver ${ }^{78}$ and were inserted into explicitly hydrated DMPC bilayers with the CHARMM-GUI ${ }^{79,80}$ membrane builder tool ${ }^{81,82}$. The bilayer included 224 DMPC molecules, with 114 in one leaflet and 110 in the second, and was hydrated with TIP3 water molecules ${ }^{83}$. The ligand forcefield was parameterized from the coordinates, and the simulation was conducted in GROMACS $^{84}$ on NMRBox virtual servers ${ }^{85}$. The simulation was conducted at $310 \mathrm{~K}$ with CHARMM36 force fields ${ }^{86-89}$ including the WYF parameter for cation $-\pi$ interactions ${ }^{90}$. The simulation was restrained by the protein-ligand ${ }^{1} \mathrm{H}-{ }^{19} \mathrm{~F}$ distances and TALOS predicted $(\phi, \psi)$ and sidechain $\chi_{1}$ torsion angles (Supplementary Fig. 2b). The restoring force for time-averaged interatomic distance restraints used was the piecewise linear force described in the online GROMACS documentation, where if $\bar{r}_{i j}$ is the time averaged distance between atoms $i$ and $j$, the 
force would be proportional to $\bar{r}_{i j}-r_{0}$ below $r_{0}$, zero between $r_{0}$ and $r_{1}$, proportional to $\bar{r}_{i j}-r_{1}$ between $r_{1}$ and $r_{2}$, and proportional to $r_{2}-r_{1}$ above $r_{2}$. Simple timeaveraged distance restraints using conservative weighting were used. The time averaging constant was set to $5 \mathrm{ps}$, and the distance restraint force constant was set to $1000 \mathrm{~kJ} /\left(\mathrm{mol}^{*} \mathrm{~nm}^{2}\right)$. Dihedral restraints were also implemented with an energy weighting of 1000 . The simulation started with a 5000-step minimization with position restraints, followed by $1.875 \mathrm{~ns}$ of equilibration over which the position restraints were progressively weakened. The production stage of the simulation was subject only to experimental constraints and was carried out in 2 fs steps for 360 and $440 \mathrm{~ns}$ in two runs (Supplementary Fig. 6b). Structural ensembles from the two trajectories were assembled by taking structures in $5 \mathrm{~ns}$ increments in the equilibrated portion of the simulation (200-400 ns for run 1, 200-360 ns for run 2) for a total of 74 structures. These structures were subjected to 5000 steps of energy minimization with position and torsion angle restraints to remove improper dihedral angles introduced by fast-timescale fluctuations. These 74 structures were scored against the original four-fold ambiguous distance constraints to select 10 structures that best agreed with the experimental data according to the sum total of distance violations (Supplementary Table 5). By chance, five of the best structures came from the first run (mean violation magnitude $0.4 \pm 0.1 \AA$, mean number of violations $=4.4 \pm 0.5$ ), while another five came from the second run (mean violation magnitude $0.4 \pm 0.2 \AA$, mean number of violations $=4.2 \pm 0.8$ ). Within each run, the ensembles are well ordered, with a mean backbone RMSD of $1.6 \pm 0.3 \AA$ for the 5 structures of run 1 , and $2.3 \pm 0.5 \AA$ for the 5 structures of run 2 . Between the two sub-ensembles, the variability was higher, at $2.9 \pm 0.5 \AA$ backbone RMSD; most of the differences are localized to the loop regions and TM4 (Supplementary Fig. 6c) where the MD simulation has few constraints: the mean backbone RMSD between the two runs for the transmembrane helices $1-3$ was $2.2 \pm 0.3 \AA$, lower than the variability within the sub-ensemble of run 2 . The water accessibility of the binding site was examined in the fully hydrated lowest-violation complexes of the bilayerprotein-ligand system after final MD energy minimization. Water molecules whose oxygen atom is within $15 \AA$ of any ligand atom and whose oxygen $z$-coordinate also lies within the top and bottom planes of the protein TM helical bundles are selected. For the pH 8.0 complex, the top and bottom $z$-planes were set to be between the F23B and G77A Ca atoms. For the pH 5.8 complex, the top and bottom z-planes were set to be between the $z$-coordinates of the Y53A and T56B Ca atoms.

Reporting summary. Further information on research design is available in the Nature Research Reporting Summary linked to this article.

\section{Data availability}

Solid-state NMR chemical shifts and distance restraints have been deposited in the Biological Magnetic Resonance Bank (BMRB) with ID number 30957. The structural coordinates have been deposited in the Protein Data Bank with accession code 7SFQ. Source data are provided with this paper.

\section{Code availability}

Python code for ${ }^{1} \mathrm{H}-{ }^{19} \mathrm{~F}$ REDOR analysis, structurally-based $\mathrm{H}-\mathrm{F}$ pair assignment, Gromacs simulations, ${ }^{19} \mathrm{~F}-{ }^{19} \mathrm{~F}$ exchange analysis, and water-edited ${ }^{1} \mathrm{H}_{-}{ }^{15} \mathrm{~N}$ analysis are available upon request to meihong@mit.edu.

Received: 24 October 2021; Accepted: 27 January 2022; Published online: 18 February 2022

\section{References}

1. Nikaido, H. Multidrug resistance in bacteria. Annu. Rev. Biochem. 78, 119-146 (2009).

2. Higgins, C. F. Multiple molecular mechanisms for multidrug resistance transporters. Nature 446, 749 (2007).

3. Yerushalmi, H., Lebendiker, M. \& Schuldiner, S. EmrE, an Escherichia coli 12$\mathrm{kDa}$ multidrug transporter, exchanges toxic cations and $\mathrm{H}+$ and is soluble in organic solvents. J. Biol. Chem. 270, 6856-6863 (1995).

4. Saleh M., Bay D. C. \& Turner R. J. Few conserved amino acids in the small multidrug resistance transporter EmrE influence drug polyselectivity. Antimicrob. Agents Chemother. 62, e00461-18 (2018).

5. Schuldiner, S. et al. Small is mighty: EmrE, a multidrug transporter as an experimental paradigm. N. Physiol. Sci. 16, 130-134 (2001).

6. Cho, M.-K., Gayen, A., Banigan, J. R., Leninger, M. \& Traaseth, N. J. Intrinsic conformational plasticity of native EmrE provides a pathway for multidrug resistance. J. Am. Chem. Soc. 136, 8072-8080 (2014).

7. Wu, C. et al. Identification of an alternating-access dynamics mutant of EmrE with impaired transport. J. Mol. Biol. 431, 2777-2789 (2019).

8. Chen, Y.-J. et al. X-ray structure of EmrE supports dual topology model. Proc. Natl Acad. Sci. USA 104, 18999 (2007).
9. Ubarretxena-Belandia, I., Baldwin, J. M., Schuldiner, S. \& Tate, C. G. Threedimensional structure of the bacterial multidrug transporter EmrE shows it is an asymmetric homodimer. EMBO J. 22, 6175-6181 (2003).

10. Kermani, A. A. et al. The structural basis of promiscuity in small multidrug resistance transporters. Nat. Commun. 11, 6064 (2020).

11. Sulavik, M. C. et al. Antibiotic susceptibility profiles of Escherichia coli strains lacking multidrug Efflux pump genes. Antimicrob. Agents Chemother. 45, 1126-1136 (2001).

12. Nishino, K., Nikaido, E. \& Yamaguchi, A. Regulation and physiological function of multidrug efflux pumps in Escherichia coli and Salmonella. Biochim. Biophys. Acta 1794, 834-843 (2009).

13. Matsumura, K., Furukawa, S., Ogihara, H. \& Morinaga, Y. Roles of multidrug efflux pumps on the biofilm formation of Escherichia coli K-12. Biocontrol Sci. 16, 69-72 (2011).

14. Bay, D. C., Stremick, C. A., Slipski, C. J. \& Turner, R. J. Secondary multidrug efflux pump mutants alter Escherichia coli biofilm growth in the presence of cationic antimicrobial compounds. Res. Microbiol. 168, 208-221 (2017).

15. Morrison, E. A., Robinson, A. E., Liu, Y. \& Henzler-Wildman, K. A. Asymmetric protonation of EmrE. J. Gen. Physiol. 146, 445-461 (2015).

16. Gayen, A., Leninger, M. \& Traaseth, N. J. Protonation of a glutamate residue modulates the dynamics of the drug transporter EmrE. Nat. Chem. Biol. 12, 141 (2016).

17. Robinson, A. E., Thomas, N. E., Morrison, E. A., Balthazor, B. M. \& HenzlerWildman, K. A. New free-exchange model of EmrE transport. Proc. Natl Acad. Sci. USA 114, E10083 (2017).

18. Thomas, N. E. et al. The C terminus of the bacterial multidrug transporter EmrE couples drug binding to proton release. J. Biol. Chem. 293, 19137-19147 (2018).

19. Hussey G. A., Thomas N. E. \& Henzler-Wildman K. A. Highly coupled transport can be achieved in free-exchange transport models. J. Gen. Physiol. 152, e201912437 (2020).

20. Tate, C. G., Ubarretxena-Belandia, I. \& Baldwin, J. M. Conformational changes in the multidrug transporter EmrE associated with substrate binding. J. Mol. Biol. 332, 229-242 (2003).

21. Morrison, E. A. et al. Antiparallel EmrE exports drugs by exchanging between asymmetric structures. Nature 481, 45 (2011).

22. Rapp, M., Granseth, E., Seppälä, S. \& von Heijne, G. Identification and evolution of dual-topology membrane proteins. Nat. Struct. Mol. Biol. 13, 112-116 (2006).

23. Lloris-Garcerá, P. P. et al. Antiparallel dimers of the small multidrug resistance protein EmrE are more stable than parallel dimers. J. Biol. Chem. 287, 26052-26059 (2012).

24. Lloris-Garcerá, P. et al. In vivo Trp scanning of the small multidrug resistance protein EmrE confirms 3D structure models. J. Mol. Biol. 425, 4642-4651 (2013).

25. Amadi, S. T., Koteiche, H. A., Mishra, S. \& McHaourab, H. S. Structure, dynamics, and substrate-induced conformational changes of the multidrug transporter EmrE in liposomes. J. Biol. Chem. 285, 26710-26718 (2010).

26. Dastvan, R., Fischer, A. W., Mishra, S., Meiler, J. \& McHaourab, H. S. Protonation-dependent conformational dynamics of the multidrug transporter EmrE. Proc. Natl Acad. Sci. USA 113, 1220-1225 (2016).

27. Kermani, A. A., Macdonald, C. B., Gundepudi, R. \& Stockbridge, R. B. Guanidinium export is the primal function of SMR family transporters. Proc. Natl Acad. Sci. USA 115, 3060-3065 (2018).

28. Gayen, A., Banigan, J. R. \& Traaseth, N. J. Ligand-induced conformational changes of the multidrug resistance transporter EmrE probed by oriented solid-state NMR spectroscopy. Angew. Chem. Int. Ed. 52, 10321-10324 (2013).

29. Dutta, S., Morrison, E. A. \& Henzler-Wildman, K. A. Blocking dynamics of the SMR transporter EmrE impairs efflux activity. Biophys. J. 107, 613-620 (2014).

30. Li J., Sae Her A. \& Traaseth N. J. Asymmetric protonation of glutamate residues drives a preferred transport pathway in EmrE. Proc. Natl Acad. Sci. USA 118, e2110790118 (2021).

31. Lehner, I. et al. The key residue for substrate transport (Glu14) in the EmrE dimer is asymmetric. J. Biol. Chem. 283, 3281-3288 (2008).

32. Ovchinnikov, V., Stone, T. A., Deber, C. M. \& Karplus, M. Structure of the EmrE multidrug transporter and its use for inhibitor peptide design. Proc. Natl Acad. Sci. USA 115, E7932 (2018).

33. Vermaas, J. V., Rempe, S. B. \& Tajkhorshid, E. Electrostatic lock in the transport cycle of the multidrug resistance transporter EmrE. Proc. Natl Acad. Sci. USA 115, E7502 (2018).

34. Roos, M., Mandala, V. S. \& Hong, M. Determination of long-range distances by fast magic-angle-spinning radiofrequency-driven $19 \mathrm{~F}-19 \mathrm{~F}$ dipolar recoupling NMR. J. Phys. Chem. B 122, 9302-9313 (2018).

35. Roos, M., Wang, T., Shcherbakov, A. A. \& Hong, M. Fast magic-angle-spinning F-19 spin exchange NMR for determining nanometer distances in proteins and pharmaceutical compounds. J. Phys. Chem. B 122, 2900-2911 (2018).

36. Shcherbakov, A. A. \& Hong, M. Rapid measurement of long-range distances in proteins by multidimensional ${ }^{13} \mathrm{C}-{ }^{19} \mathrm{~F}$ REDOR NMR under fast magicangle spinning. J. Biomol. NMR 71, 31-43 (2018). 
37. Shcherbakov, A. A., Mandala, V. S. \& Hong, M. High-sensitivity detection of nanometer $1 \mathrm{H}-19 \mathrm{~F}$ distances for protein structure determination by $1 \mathrm{H}-$ detected fast MAS NMR. J. Phys. Chem. B 123, 4387-4391 (2019).

38. Shcherbakov, A. A., Roos, M., Kwon, B. \& Hong, M. Two-dimensional 19F-13C correlation NMR for 19F resonance assignment of fluorinated proteins. J. Biomol. NMR 74, 193-204 (2020).

39. Shcherbakov, A. A. et al. Structure and dynamics of the drug-bound bacterial transporter EmrE in lipid bilayers. Nat. Commun. 12, 172 (2021).

40. Yerushalmi, H. \& Schuldiner, S. An essential glutamyl residue in EmrE, a multidrug antiporter from Escherichia coli. J. Biol. Chem. 275, 5264-5269 (2000).

41. Yerushalmi, H., Mordoch, S. S. \& Schuldiner, S. A single carboxyl mutant of the multidrug transporter EmrE is fully functional. J. Biol. Chem. 276, 12744-12748 (2001).

42. Muth, T. R. \& Schuldiner, S. A membrane-embedded glutamate is required for ligand binding to the multidrug transporter EmrE. EMBO J. 19, 234-240 (2000).

43. Brill, S., Falk, O. S. \& Schuldiner, S. Transforming a drug/H+ antiporter into a polyamine importer by a single mutation. Proc. Natl Acad. Sci. USA 109, 16894-16899 (2012).

44. Brill, S., Sade-Falk, O., Elbaz-Alon, Y. \& Schuldiner, S. Specificity determinants in small multidrug transporters. J. Mol. Biol. 427, 468-477 (2015).

45. Wang, J., Rath, A. \& Deber, C. M. Functional response of the small multidrug resistance protein EmrE to mutations in transmembrane helix 2. FEBS Lett. 588, 3720-3725 (2014).

46. Elbaz, Y., Salomon, T. \& Schuldiner, S. Identification of a glycine motif required for packing in EmrE, a multidrug transporter from Escherichia coli. J. Biol. Chem. 283, 12276-12283 (2008).

47. Wilks, J. C. \& Slonczewski, J. L. pH of the cytoplasm and periplasm of Escherichia coli: Rapid measurement by green fluorescent protein fluorimetry. J. Bacteriol. 189, 5601-5607 (2007).

48. Slonczewski, J. L., Rosen, B. P., Alger, J. R. \& Macnab, R. M. pH homeostasis in Escherichia coli: Measurement by 31P nuclear magnetic resonance of methylphosphonate and phosphate. Proc. Natl Acad. Sci. USA 78, 6271-6275 (1981).

49. Morrison, E. A. \& Henzler-Wildman, K. A. Reconstitution of integral membrane proteins into isotropic bicelles with improved sample stability and expanded lipid composition profile. Biochim. Biophys. Acta 1818, 814-820 (2012).

50. Hong, M. \& Schmidt-Rohr, K. Magic-angle-spinning NMR techniques for measuring long-range distances in biological macromolecules. Acc. Chem. Res. 46, 2154-2163 (2013).

51. Morrison, E. A. \& Henzler-Wildman, K. A. Transported substrate determines exchange rate in the multidrug resistance transporter EmrE. J. Biol. Chem. 289, 6825-6836 (2014).

52. Delaglio, F. et al. NMRPipe: A multidimensional spectral processing system based on UNIX pipes. J. Biomol. NMR 6, 277-293 (1995).

53. Vranken, W. F. et al. The CCPN data model for NMR spectroscopy: Development of a software pipeline. Proteins: Struct., Funct., Bioinform. 59, 687-696 (2005).

54. Shrager, R. I., Cohen, J. S., Heller, S. R., Sachs, D. H. \& Schechter, A. N. Mathematical models for interacting groups in nuclear magnetic resonance titration curves. Biochemistry 11, 541-547 (1972).

55. McIntosh, L. P. et al. Dissecting electrostatic interactions in Bacillus circulans xylanase through NMR-monitored pH titrations. J. Biomol. NMR 51, 5-19 (2011).

56. Cavanagh J., Fairbrother W. J., Palmer A. G. III \& Skelton N. J. Protein NMR Spectroscopy: Principles and Practice (Academic Press, 1996).

57. Vasa, S. K., Singh, H., Rovó, P. \& Linser, R. Dynamics and interactions of a 29 kDa human enzyme studied by solid-state NMR. J. Phys. Chem. Lett. 9 1307-1311 (2018).

58. Baldus, M., Petkova, A. T., Herzfeld, J. \& Griffin, R. G. Cross polarization in the tilted frame: Assignment and spectral simplification in heteronuclear spin systems. Mol. Phys. 95, 1197-1207 (1998).

59. Verel, R., Ernst, M. \& Meier, B. H. Adiabatic dipolar recoupling in solid-state NMR: The DREAM scheme. J. Magn. Reson. 150, 81-99 (2001).

60. Shaka, A. J., Keeler, J., Frenkiel, T. \& Freeman, R. An improved sequence for broadband decoupling: WALTZ-16. J. Magn. Reson. 52, 335-338 (1983).

61. Zhou, D. H. \& Rienstra, C. M. High-performance solvent suppression for proton detected solid-state NMR. J. Magn. Reson. 192, 167-172 (2008).

62. Dürr, H. N., Grage, S. L., Witter, R. \& Ulrich, A. S. Solid state ${ }^{19}$ F NMR parameters of fluorine-labeled amino acids. Part I: Aromatic substituents. J. Magn. Reson. 191, 7-15 (2008).

63. Walt, Svd, Colbert, S. C. \& Varoquaux, G. The NumPy array: A structure for efficient numerical computation. Comput. Sci. Eng. 13, 22-30 (2011).

64. Helmus, J. J. \& Jaroniec, C. P. Nmrglue: An open source Python package for the analysis of multidimensional NMR data. J. Biomol. NMR 55, 355-367 (2013).

65. Lee, W., Tonelli, M. \& Markley, J. L. NMRFAM-SPARKY: Enhanced software for biomolecular NMR spectroscopy. Bioinformatics 31, 1325-1327 (2014).

66. Hunter, J. D. Matplotlib: A 2D graphics environment. Comput. Sci. Eng. 9, 90-95 (2007).
67. Shen, Y. \& Bax, A. Protein backbone and sidechain torsion angles predicted from NMR chemical shifts using artificial neural networks. J. Biomol. NMR 56, 227-241 (2013)

68. Virtanen, P. et al. SciPy 1.0: Fundamental algorithms for scientific computing in Python. Nat. Methods 17, 261-272 (2020).

69. Öster, C., Kosol, S. \& Lewandowski, J. R. Quantifying microsecond exchange in large protein complexes with accelerated relaxation dispersion experiments in the solid state. Sci. Rep. 9, 11082 (2019).

70. Bak, M., Rasmussen, J. T. \& Nielsen, N. C. SIMPSON: A general simulation program for solid-state NMR spectroscopy. J. Magn. Reson. 147, 296-330 (2000).

71. Bak, M. \& Nielsen, N. C. REPULSION, a novel approach to efficient powder averaging in solid-state NMR. J. Magn. Reson. 125, 132-139 (1997).

72. Mandala, V. S., Liao, S. Y., Kwon, B. \& Hong, M. Structural basis for asymmetric conductance of the influenza M2 proton channel investigated by solid-state NMR spectroscopy. J. Mol. Biol. 429, 2192-2210 (2017).

73. Dregni, A. J., Duan, P. \& Hong, M. Hydration and dynamics of full-length Tau amyloid fibrils investigated by solid-state nuclear magnetic resonance. Biochemistry 59, 2237-2248 (2020).

74. Mandala, V. S., Loftis, A. R., Shcherbakov, A. A., Pentelute, B. L. \& Hong, M. Atomic structures of closed and open influenza B M2 proton channel reveal the conduction mechanism. Nat. Struct. Mol. Biol. 27, 160-167 (2020).

75. van Zundert, G. C. P. et al. The HADDOCK2.2 Web Server: User-friendly integrative modeling of biomolecular complexes. J. Mol. Biol. 428, 720-725 (2016).

76. de Vries, S. J., van Dijk, M. \& Bonvin, A. M. J. J. The HADDOCK web server for data-driven biomolecular docking. Nat. Protoc. 5, 883-897 (2010).

77. Sharoni, M., Steiner-Mordoch, S. \& Schuldiner, S. Exploring the binding domain of EmrE, the smallest multidrug transporter. J. Biol. Chem. 280, 32849-32855 (2005)

78. Lomize, M. A., Pogozheva, I. D., Joo, H., Mosberg, H. I. \& Lomize, A. L. OPM database and PPM web server: Resources for positioning of proteins in membranes. Nucleic Acids Res. 40, D370-D376 (2012).

79. Jo, S., Kim, T., Iyer, V. G. \& Im, W. CHARMM-GUI: A web-based graphical user interface for CHARMM. J. Comput. Chem. 29, 1859-1865 (2008).

80. Lee, J. et al. CHARMM-GUI input generator for NAMD, GROMACS, AMBER, OpenMM, and CHARMM/OpenMM simulations using the CHARMM36 additive force field. J. Chem. Theory Comput. 12, 405-413 (2016).

81. Jo, S., Kim, T. \& Im, W. Automated builder and database of protein/ membrane complexes for molecular dynamics simulations. PLoS One 2, e880 (2007).

82. Wu, E. L. et al. CHARMM-GUI membrane builder toward realistic biological membrane simulations. J. Comput. Chem. 35, 1997-2004 (2014).

83. Mark, P. \& Nilsson, L. Structure and dynamics of the TIP3P, SPC, and SPC/E water models at 298 K. J. Phys. Chem. A 105, 9954-9960 (2001)

84. Berendsen, H. J. C., van der Spoel, D. \& van Drunen, R. GROMACS: A message-passing parallel molecular dynamics implementation. Comput. Phys. Commun. 91, 43-56 (1995)

85. Maciejewski, M. W. et al. NMRbox: A resource for biomolecular NMR computation. Biophys. J. 112, 1529-1534 (2017).

86. Jorgensen, W. L., Chandrasekhar, J., Madura, J. D., Impey, R. W. \& Klein, M. L. Comparison of simple potential functions for simulating liquid water. $J$. Chem. Phys. 79, 926-935 (1983).

87. MacKerell, A. D. et al. All-atom empirical potential for molecular modeling and dynamics studies of proteins. J. Phys. Chem. B 102, 3586-3616 (1998).

88. Klauda, J. B. et al. Update of the CHARMM all-atom additive force field for lipids: Validation on six lipid types. J. Phys. Chem. B 114, 7830-7843 (2010).

89. Huang, J. et al. CHARMM $36 \mathrm{~m}$ : An improved force field for folded and intrinsically disordered proteins. Nat. Methods 14, 71-73 (2017).

90. Khan, H. M., MacKerell, A. D. \& Reuter, N. Cation- $\pi$ interactions between methylated ammonium groups and tryptophan in the CHARMM36 additive force field. J. Chem. Theory Comput. 15, 7-12 (2019).

\section{Acknowledgements}

This work is supported by NIH grants GM095839 to K.A. H-W, GM088204 to M.H., and the MIT School of Science Camplan Fund to A.A.S. and M.H. The study made use of NMR spectrometers at the National Magnetic Resonance Facility at Madison, supported by NIH grant P41 GM103399 and P41 RR002301; the Center for Magnetic Resonance, supported by P41 GM132079; and the NMRbox, supported by P41 GM111135. Equipment was purchased with funds from the University of Wisconsin-Madison, NIH (S10RR02781, S10RR08438, S10RR023438, S10RR025062, S10RR029220), the NSF (DMB-8415048, OIA-9977486, BIR-9214394), and the USDA.

\section{Author contributions}

M.H. and K.A.H.-W. designed the experiments. A.A.S. conducted the solid-state NMR experiments, data analysis, and structure calculation; P.S. purified the protein and 
prepared the membrane samples; A.J.D. contributed to the solid-state NMR experiments and data analysis. All authors discussed the results and wrote the paper.

\section{Competing interests}

The authors declare no competing interests.

\section{Additional information}

Supplementary information The online version contains supplementary material available at https://doi.org/10.1038/s41467-022-28556-6.

Correspondence and requests for materials should be addressed to Mei Hong.

Peer review information Nature Communications thanks Markus Weingarth and the other, anonymous, reviewer(s) for their contribution to the peer review of this work. Peer reviewer reports are available.

Reprints and permission information is available at http://www.nature.com/reprints
Publisher's note Springer Nature remains neutral with regard to jurisdictional claims in published maps and institutional affiliations.

(c) (i) Open Access This article is licensed under a Creative Commons Attribution 4.0 International License, which permits use, sharing, adaptation, distribution and reproduction in any medium or format, as long as you give appropriate credit to the original author(s) and the source, provide a link to the Creative Commons license, and indicate if changes were made. The images or other third party material in this article are included in the article's Creative Commons license, unless indicated otherwise in a credit line to the material. If material is not included in the article's Creative Commons license and your intended use is not permitted by statutory regulation or exceeds the permitted use, you will need to obtain permission directly from the copyright holder. To view a copy of this license, visit http://creativecommons.org/ licenses/by/4.0/.

(C) The Author(s) 2022 